Copyright

by

$\mathrm{Bo} \mathrm{Hu}$

2002 
The Dissertation Committee for $\mathrm{Bo} \mathrm{Hu}$

certifies that this is the approved version of the following dissertation:

\section{Chaotic Scattering and the Magneto-Coulomb Map}

Committee:

Wendell Horton, Supervisor

Richard Hazeltine

Linda Reichl

Tomio Petrosky

Gary Hallock 


\title{
Chaotic Scattering and the Magneto-Coulomb Map
}

\author{
by
}

Bo $\mathrm{Hu}$, M.A.

\author{
DISSERTATION \\ Presented to the Faculty of the Graduate School of \\ The University of Texas at Austin \\ in Partial Fulfillment \\ of the Requirements \\ for the Degree of \\ DOCTOR OF PHILOSOPHY
}

THE UNIVERSITY OF TEXAS AT AUSTIN

August 2002 
Dedicated to my wife Tong. 


\section{Acknowledgments}

I wish to thank my supervisor Professor Wendell Horton for kindly accepting me and supervising me in my studies. I also wish to thank Dr. Tomio Petrosky for supervising me and always encouraging me in my research. I have also learned very much from Dr. Ping Zhu in the fields of plasma and computing. I have received generous help and good suggestions from many people, e.g., Glenn Bateman, James Callen, Francesca Bombarda, Linda Sugiyama, Bruno Coppi, Franco Porcelli, Gary Hallock, Philip Watson, Tuong Hoang, Charles Chiu, Joseph Godino. I am also grateful to IFS faculty and staff. I finally have to thank my wife for working hard to support the family, even though she does not like to work. 


\title{
Chaotic Scattering and the Magneto-Coulomb Map
}

\author{
Publication No. \\ Bo Hu, Ph.D. \\ The University of Texas at Austin, 2002
}

Supervisor: Wendell Horton

In Chapter 1, a non-relativistic classical electron scattering by a fixed ion in a uniform magnetic field is studied. The system is non-integrable, and there is chaotic scattering for certain regimes of initial conditions. A two dimensional discrete map is derived from the equation of motion. The map exhibits different types of motion by changing the parameters which characterize the initial condition. The fractal structure is observed. The width of the chaotic scattering region is estimated. A certain class of plasma enviroments is suggested, where the chaotic scattering may have an important role.

In Chapter 2, a fast plasma current ramping approach to achieve reversed magnetic shear confinement enhancement and peaked density profiles for Ignitor tokamak machine is studied with the BALDUR simulation code. Improved confinement regimes require peaked density profiles. Peaked density profiles are necessary for supressing the turbulence and optimizing the fusion power of a tokamak plasma. Previous transport simulations used either 
density profiles with fixed peaking shape or obtained flat density profiles for Ignitor. Reversed magnetic shear may supress the transport coefficients and create the internal transport barrier. Together with controlled gas puffing, the transport barrier produces peaked density profiles. Reversed magnetic shear also helps to delay the onset of the sawtooth. Such a programmed Ohmic heating scheme is demonstrated to be an effective way to achieve ignition in a burning D-T plasma. 


\section{Table of Contents}

Acknowledgments $\quad$ v

Abstract vi vi vis

List of Tables $\quad$ x

List of Figures

Chapter 1. Chaotic Scattering and the Magneto-Coulomb Map 1

1.1 Introduction ................. 1

1.2 Dimensionless units . . . . . . . . . . . . . . 4

1.3 Derivation of map . . . . . . . . . . . . . . 6

1.4 Comparison of map with numerical integration . . . . . . . . 13

1.5 Phase space plots . . . . . . . . . . . . . 17

1.6 Classification of motion . . . . . . . . . . . . . 21

1.7 Fractal structure . . . . . . . . . . . . . . 24

1.8 Width of chaotic scattering region . . . . . . . . . . 26

1.9 Radiation energy loss ................ 27

1.10 Anomalous transport .................. 28

Chapter 2. Reversed Magnetic Shear Confinement for Ignitor 31

2.1 Introduction to tokamaks . . . . . . . . . . . . . 31

2.2 Introduction to Ignitor . . . . . . . . . . . . . . . . . . . . . . . . . . . . . . 35

2.3 Problems and motivation . . . . . . . . . . . 36

2.4 Tokamak plasma transport code BALDUR . . . . . . . . . 39

2.5 Ohmic heating and current penetration . . . . . . . . . . . 40

2.6 Tokamak circuit . . . . . . . . . . . . . . 43

2.7 Alpha heating . . . . . . . . . . . . . 46

2.8 Thermonuclear gain parameter . . . . . . . . . 48

viii 
2.9 Edge gas puffing and recycling . . . . . . . . . . . 48

2.10 Transport model JETTO and simulation scheme . . . . . . 51

2.11 Simulation results ................ 56

2.12 Summary . . . . . . . . . . . . . . 62

2.13 Reversed shear experiments . . . . . . . . . . . 64

2.14 MHD stability . . . . . . . . . . . . . 65

$\begin{array}{ll}\text { Appendix } & 68\end{array}$

Appendix A. Improved Maps for Anomalous Transport $\quad 69$

A.1 Multi-ion map for anomalous transport . . . . . . . . . 69

A.2 Anomaly in transport cofficients . . . . . . . . . . . 70

A.3 Derivation of convergent map ........... . 71

$\begin{array}{ll}\text { Bibliography } & 74\end{array}$

$\begin{array}{ll}\text { Vita } & 78\end{array}$ 


\section{List of Tables}

1.1 Representative plasmas where chaotic scattering is predicted. 29

2.1 Ignitor compared with some other proposed tokamak machines 36

2.2 Global parameters for Ignitor simulation. . . . . . . . . 53 


\section{List of Figures}

1.1 Initial sensitivity for chaotic scattering. The dot denotes the ion. Initial conditions are (a) $b=1.02$ and (b) $b=1.03$ with the same $z(t=0)=-10$ and $\boldsymbol{v}(t=0)=1.0 \hat{\boldsymbol{z}}$. . . . . .

1.2 A typical orbit of the chaotic scattering. The dot denotes the ion. The electron starts from the left to the ion $(z=-10)$ with a velocity $v=10$ in the $z$ direction, and an impact parameter $b=0.0096$. After the electron is scattered by a nearly $90^{\circ}$ angle, the guiding center is moving in a nearly straight line parallel to the magnetic field, and the electron is doing a nearly circular motion in the $x y$ plane. . . . . . . . . . . . .

1.3 Variables in the map. . . . . . . . . . . . . .

1.4 The comparsion between the map and the numerical integration for $\left(z, v_{z}\right)$ vs $t$. The initial condition is $z^{0}=1.5, v_{z}^{0}=0.4, v=10$ and $d=10$. The cyclotron period is $2 \pi$. . . . . . .

1.5 The comparison of the phase space $\left(\varepsilon_{z}, \tau\right)$ plots between the map and the numerical integration for the trapped chaotic motion near scattering regime. The initial condition is $z^{0}=1.1, v_{z}^{0}=0$,

1.6 Trapped chaotic motion in $\left(\varepsilon_{z}, \tau\right)$ near scattering regime. . . 18

1.7 Phase space plot for scattering motion from map. . . . . . . . 19

1.8 Islands of the trapped motion inside the scattering region. . . 20

1.9 Four types of orbits, $v=10$. . . . . . . . . . . . 22

1.10 Four types of motion. . . . . . . . . . . . 23

1.11 Fractal structure obtained from the map in $v_{z}^{\mathrm{f}}$ vs $v_{z}^{0}$ for initial conditions $v_{z}^{0} \in$ (a) $[0.2,0.25]$ and (b) $[0.2,0.2001], z^{0}=1.5$, $v=10$ and $d=10$. . . . . . . . . . .

1.12 Fractal structure obtained from the map in $n_{z}$ vs $v_{z}^{0}$ for initial conditions $v_{z}^{0} \in(\mathrm{a})[0.2,0.25]$ and (b) $[0.2,0.2001], z^{0}=1.5$, $v=10$ and $d=10 . \ldots \ldots \ldots$

1.13 Width $\delta b$ of chaotic scattering region in the impact parameter $b$ as a function of velocity $v \ldots . . . . . . .$. 
2.1 Time history of a) plasma current $I_{\mathrm{p}}$, b) volume-average density $\left.\left\langle n_{\mathrm{e}}\right\rangle, \mathrm{c}\right)$ Ohmic power $P_{\mathrm{oh}}$ and $\alpha$ power $P_{\alpha}$, and d) confinement time $\tau_{\mathrm{E}}$ in the reference simulation run f01 with peaked density

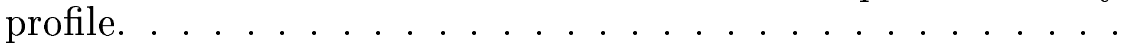

2.2 Comparison of time evolutions of density profile peakness $n_{\mathrm{e}}(0) /\left\langle n_{\mathrm{e}}\right\rangle$ for two simulation runs with different current ramping rates, i.e., f01 with fast rate and a010 with slow rate. . . . . . . 56

2.3 Radial profiles of a) plasma current density $j$, b) safety factor $q$, c) particle diffusivity $D_{i}$, d) electron density $n_{\mathrm{e}}$, e) ion thermal conductivity $\chi_{i}$, and $\mathrm{f}$ ) ion temperature $T_{i}$, at six time slices $t=0,1,2,3,4$ and $4.6 \mathrm{~s}$ in the reference simulation run f01. .

2.4 Radial profiles of a) plasma current density $j$, b) safety factor $q$, c) particle diffusivity $D_{i}$, d) electron density $n_{\mathrm{e}}$, e) ion thermal conductivity $\chi_{i}$, and $\mathrm{f}$ ) ion temperature $T_{i}$, at six time slices $t=0,1,2,3,4$ and $5 \mathrm{~s}$ in the reference simulation run a010. . .

2.5 Time traces of alpha heating power $P_{\alpha}$ and the total confinement power loss $P_{\mathrm{L}}$ in the two simulation runs a010 and f01.

2.6 Comparison for time evolution of the plasma current between simulation runs $\mathrm{f} 01$ and $a 010 . \ldots \ldots \ldots$

2.7 Comparison for time evolution of the position of $q=1$ surface between simulation runs f01 and a010. . . . . . . . .

2.8 Current density $j$, safety factor $q$ and electron density $n_{\mathrm{e}}$ profiles at six time slices $t=0,1,2,3,4,5 \mathrm{~s}$. Results from BALDUR simulation with multi-mode model. . . . . . . . . .

A.1 Model for multi-ion map. . . . . . . . . . . . .

A.2 $z^{0}=\left(\right.$ Improved Map)1.75, (Numerical Integration) $1.86, v_{z}^{0}=0$, $v=1, d=3 \ldots \ldots \ldots \ldots \ldots$ 


\section{Chapter 1}

\section{Chaotic Scattering and the Magneto-Coulomb Map}

The existing ohmic heating model takes the electron-ion scattering to be Rutherford scattering. The original goal of this work was to find the possible corrections to the existing ohmic heating model for high-magnetic-field tokamaks like Ignitor.

In Chapter 2 Ohmic heating and the control of plasma confinement in the high field pulsed ignition experiment called Ignitor is explored complex transport simulations.

\section{$1.1 \quad$ Introduction}

In this chapter, the problem of a non-relativistic classical electron scattering by a fixed ion in a uniform magnetic field is considered. This system is non-integrable, hence one can expect the appearance of the chaotic scattering for certain class of initial conditions. Indeed, this has been noticed by Delos et al. [1] and analyzed later by Schmidt et al. [2] (also see [3-8] for the trapped motion of the electron and for the classical case as well as the quantum case). So far, all analyses were based on the continuous flow generated by the equa- 
tion of motion. However, as shown below, there are many cyclotron periods in a single scattering during the electron-ion interaction time interval. As a result, the numerical integration of the equation of motion for the continuous flow is very time-consuming and the predictions for long time behavior become unstable, which is the typical case of the chaotic scattering. For this reason, it is desirable to derive a discrete map of the chaotic scattering.

In order to get a picture of the chaotic scattering, let us first consider the case that an incoming electron with an initial velocity parallel to the magnetic field in the $z$ direction (see Fig. 1.1). More general initial conditions will be considered later. Suppose the electron is scattered by a nearly $90^{\circ}$ angle, and it starts doing cyclotron motion in the neighborhood of the ion. Due to the attractive force from the ion, while doing circular motion in the $x y$ plane, the electron oscillates along the $z$ direction, and eventually escapes in the forward or backward direction. In the phase space, there is a domain where the scattered electron is very sensitive with respect to a slight change of the initial condition. In Fig. 1.1, the two electron orbits have been numerically integrated from the equation of motion (1.10) given later. Both of the electrons have the same dimensionless initial velocity $\boldsymbol{v}(t=0)=1.0 \hat{\boldsymbol{z}}$ and the dimensionless position $z(t=0)$, but a slightly different dimensionless impact parameters, i.e., $b=1.02$ and $b=1.03$ respectively. The normalization will be introduced in Section 1.3. Eventually the electron in Fig. 1.1(a) is scattered backward, while the electron in Fig. 1.1(b) is scattered forward. These examples show that, indeed, many cyclotron periods appear before the oscillation in the $z$ 
(a)

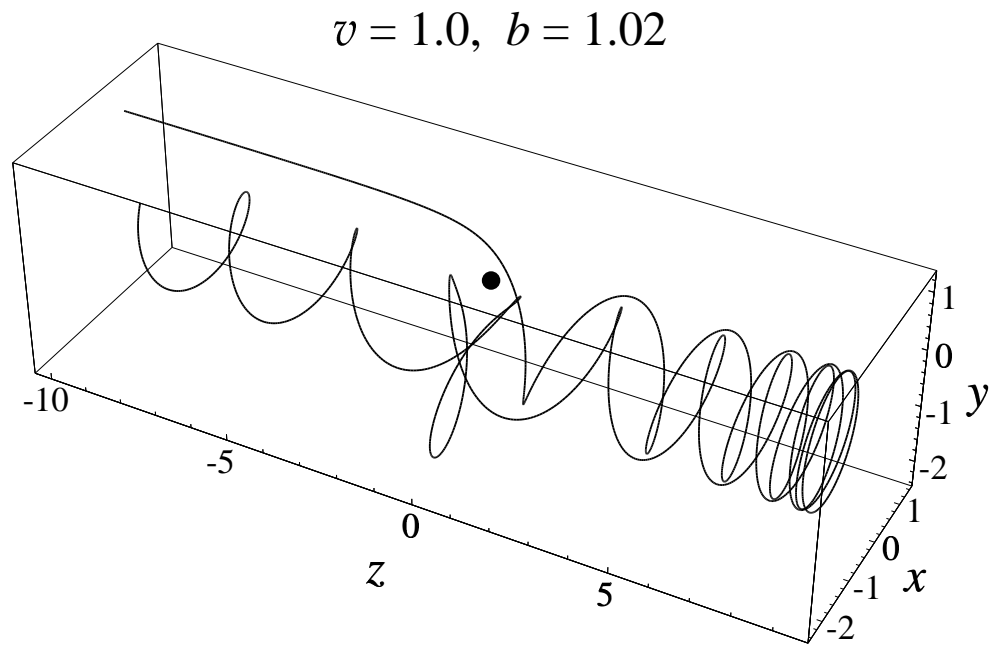

(b)

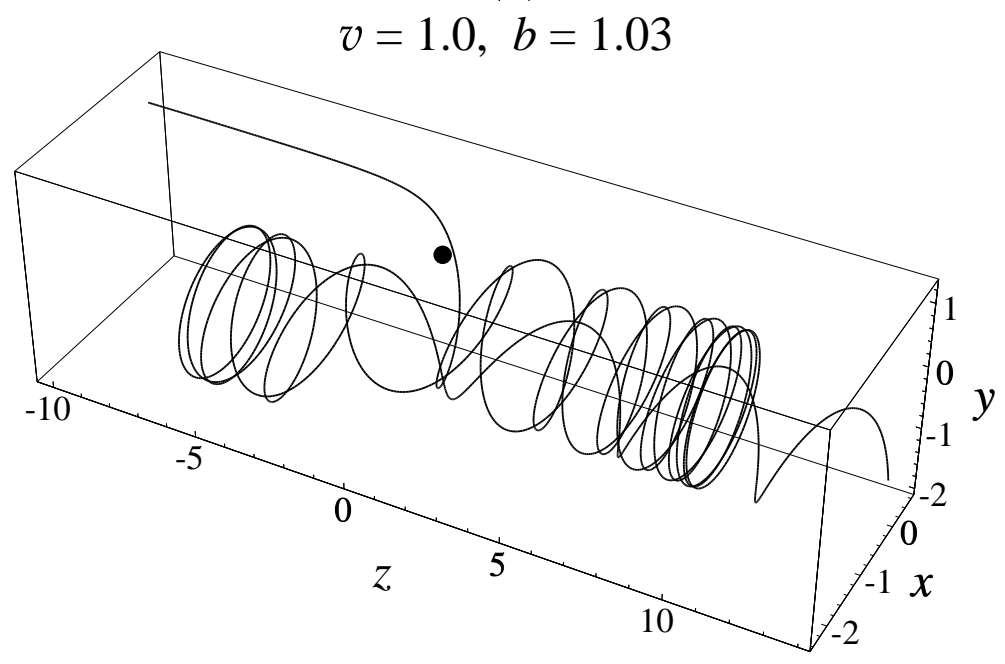

Figure 1.1: Initial sensitivity for chaotic scattering. The dot denotes the ion. Initial conditions are (a) $b=1.02$ and (b) $b=1.03$ with the same $z(t=0)=-10$ and $\boldsymbol{v}(t=0)=1.0 \hat{\boldsymbol{z}}$. 
direction ends.

The structure of this chapter is as follows. In Section 1.2 a dimensionless formalism is introduced. In Section 1.3 a two-dimensional map is derived. In Section 1.4 the map is compared with the numerical integration, and the agreement is excellent. In Section 1.5, the phase plots obtained from the map are presented for a some situations. In Section 1.6 a parameter $d$ dependence of motion is discussed, where $d$ is defined as the distance of the guiding center line of the electron orbit from the ion. In Section 1.7, the fractal structure is presented. In Section 1.8 the size of the chaotic scattering region is estimated in the impact parameter $b$ as a function of the velocity $v$ of the electron. In Section1.9, the radiation loss due to electron acceleration is estimated. In Section1.10, a comment is given on the plasma enviroments, where the chaotic scattering may play an important role.

\subsection{Dimensionless units}

Consider an electron in a Coulomb potential of a fixed ion with a charge $Z e$ at the origin together with a uniform magnetic field $\boldsymbol{B}=B \hat{\boldsymbol{z}}$. The Hamiltonian in the cylindrical coordinates $(\tilde{r}, \tilde{\phi}, \tilde{z})$ and the momenta $\left(\tilde{P}_{r}, \tilde{P}_{\phi}, \tilde{P}_{z}\right)$ is

$$
\tilde{H}=\frac{\tilde{P}_{r}^{2}}{2 m_{\mathrm{e}}}+\frac{\tilde{P}_{z}^{2}}{2 m_{\mathrm{e}}}+\frac{1}{2 m_{\mathrm{e}}}\left(\frac{\tilde{P}_{\phi}}{\tilde{r}}+\frac{\omega_{\mathrm{c}} m_{\mathrm{e}} \tilde{r}}{2}\right)^{2}-\frac{1}{4 \pi \epsilon_{0}} \frac{Z e^{2}}{\sqrt{\tilde{r}^{2}+\tilde{z}^{2}}}
$$


where the variables with dimensions are denoted by the tildes. There is a characteristic time scale, i.e., the cyclotron frequency

$$
\omega_{\mathrm{c}}=\frac{e B}{m_{\mathrm{e}}},
$$

which can be used to introduce the dimensionless time $t$,

$$
t=\omega_{\mathrm{c}} \tilde{t}
$$

A space scale $r_{0}$ is defined as the radius of a circular Keplerian motion such that the Keplerian frequency is equal to the cyclotron frequency,

$$
\frac{1}{r_{0}^{3 / 2}} \sqrt{\frac{Z e^{2}}{4 \pi \epsilon_{0} m_{\mathrm{e}}}}=\omega_{\mathrm{c}}, \quad r_{0}=\left(\frac{Z m_{\mathrm{e}}}{4 \pi \epsilon_{0} B^{2}}\right)^{1 / 3},
$$

and the length is normalized as

$$
\boldsymbol{r}=\tilde{\boldsymbol{r}} / r_{0}
$$

In this system there are two invariants of motion, i.e., the energy and the angular momentum. These invariants are also normalized as

$$
P_{\phi}=\frac{\tilde{P}_{\phi}}{m_{\mathrm{e}} \omega_{\mathrm{c}}^{2} r_{0}}, \quad \mathcal{E}=\frac{\tilde{\mathcal{E}}}{m_{\mathrm{e}} \omega_{\mathrm{c}}^{2} r_{0}^{2}} .
$$

We have

$$
\begin{aligned}
P_{\phi} & =(x \dot{y}-y \dot{x})-\frac{1}{2}\left(x^{2}+y^{2}\right), \\
\mathcal{E} & =\frac{1}{2}\left(\dot{x}^{2}+\dot{y}^{2}+\dot{z}^{2}\right)-\frac{1}{\left(x^{2}+y^{2}+z^{2}\right)^{1 / 2}} .
\end{aligned}
$$

The dimensionless Hamiltonian is given by

$$
H=\frac{P_{r}^{2}}{2}+\frac{P_{z}^{2}}{2}+\frac{1}{2}\left(\frac{P_{\phi}}{r}+\frac{r}{2}\right)^{2}-\frac{1}{\sqrt{r^{2}+z^{2}}} .
$$


This leads to the equation of motion,

$$
\ddot{\boldsymbol{r}}=\dot{\boldsymbol{r}} \times \hat{\boldsymbol{z}}-\frac{\hat{\boldsymbol{r}}}{r^{2}} .
$$

There is no parameter in the equation of motion, hence the only controllable factor is the initial condition in the dimensionless units. We note that the equation of motion (1.10) has a similar structure for the restricted three-body problem in celestial mechanics where the Coriolis force corresponds to the magnetic force $[6,9]$. For this case the chaotic scattering in the vicinity of the parabolic motion has been noticed [10].

\subsection{Derivation of map}

For the scattering problem, both the incoming and the outgoing states of the electron are generally helical cyclotron orbits (see, e.g., Fig. 1.2). In order to get a map in a simple form that exhibits a chaotic behavior, we restrict our interest to the case where the Coulomb force may be treated as a small perturbation to the magnetic force. One can easily expect that this can be achieved if the two conditions,

$$
v_{z} \ll v \quad \text { and } \quad v \gg 1 \text {, }
$$

are satisfied during the cyclotron motion, where $v_{z}$ is the component of $\boldsymbol{v}$ parallel to $z$. Indeed, the first condition $v_{z} \ll v$ gives $v_{\perp} \approx v$, where $v_{\perp}$ is the component of $\boldsymbol{v}$ perpendicular to $z$. This condition gives the dimensionless cyclotron radius $\rho_{\mathrm{e}} \approx v$. Due to the second condition $v \gg 1$, the electron 


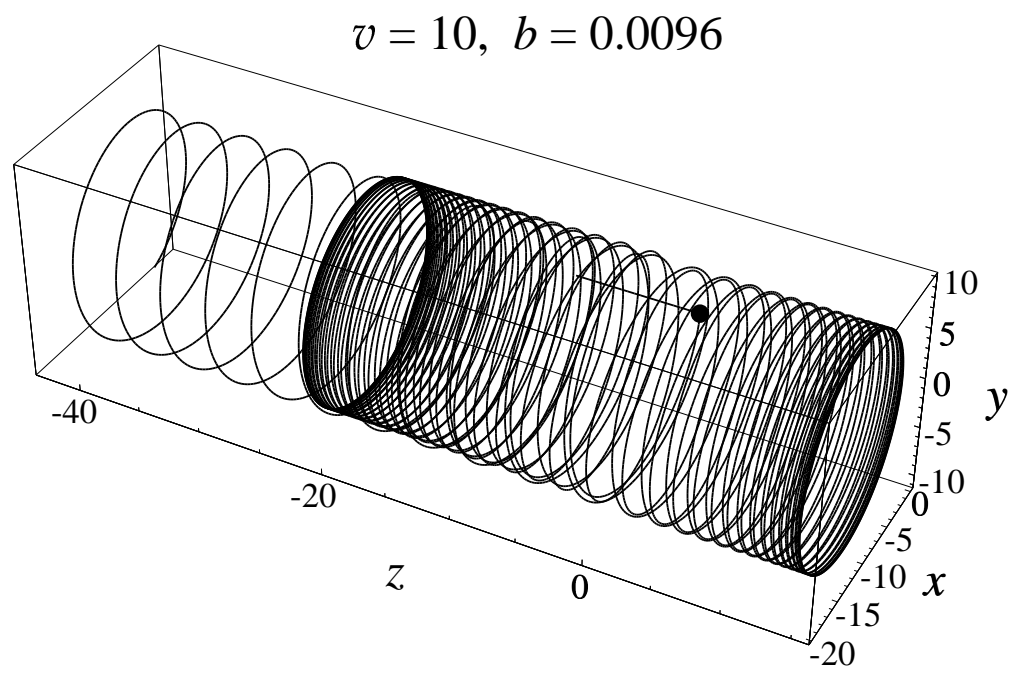

Figure 1.2: A typical orbit of the chaotic scattering. The dot denotes the ion. The electron starts from the left to the ion $(z=-10)$ with a velocity $v=10$ in the $z$ direction, and an impact parameter $b=0.0096$. After the electron is scattered by a nearly $90^{\circ}$ angle, the guiding center is moving in a nearly straight line parallel to the magnetic field, and the electron is doing a nearly circular motion in the $x y$ plane.

spends almost all the time far from the ion, and the cyclotron motion dominates during each cyclotron period. If the relation $v_{z} \ll v$ is satisfied, then the magnitude of the magnetic force is approximately $v \gg 1$. The magnitude of the electric force is $\frac{1}{z^{2}+r_{\perp}^{2}}$, where $r_{\perp}$ is the distance between the electron and the ion in the $x y$ plane. The variation of $r_{\perp}$ has the order of magnitude of $v$ during one cyclotron period. Thus most of the time, the magnetic force is dominant. Thus the Coulomb force may be treated as a small perturbation. Then again due to the first condition of (1.11), the motion of the guiding center of the electron is so slow that the Coulomb force may give a long-time 
resonance effect that leads to a chaotic motion.

On the other hand, if the relation $v \lesssim 1$ is satisfied, then after being scattered, the electron is confined in the neighborhood of the ion by the magnetic field for a time period comparable to or longer than one cyclotron period. Throughout this confinement period, the magnitude of the electric force is comparable to that of the magnetic force. In this case the electric field and the magnetic field are strongly coupled, and the electric field cannot be treated as a small perturbation.

Moreover, one may expect that if the condition (1.11) is satisfied, the distance between the guiding center line and the ion remains nearly constant during the scattering for almost all initial conditions. Indeed the numerical integration has verified this prediction. If during one cyclotron period the changes in $v_{z}$ and $z$ are not big, we can assume constant $v_{z}$ and $z$ in the cyclotron period in order to calculate the maximum and the minimum values of $r$. We find that $r_{\min }$ and $r_{\max }$ are nearly constant, i.e., independent of $v_{z}$ and $z$, if $v_{z} \ll v$. Therefore let $d$ be the distance between the ion and the guiding center line, and $d$ is adiabatic invariant. In Fig. 1.2, we show a typical orbit of the chaotic scattering that satisfies the condition (1.11). We see that the motion in the $x y$ plane is nearly circular and the guiding center is moving in a nearly straight line parallel to the magnetic field. The chance for the electron to have a large-angle scattering by the ion becomes smaller when $v$ becomes larger. This is also obvious by saying that a plasma with a high temperature havs a lower collisionality. Large-angle scattering with 
Rutherford approximation gives $v^{2} b \lesssim 1$. If assume $v_{z} \sim 1$, then the chance for the electron to have large-angle scattering is approximately $\frac{1}{v^{2}}$.
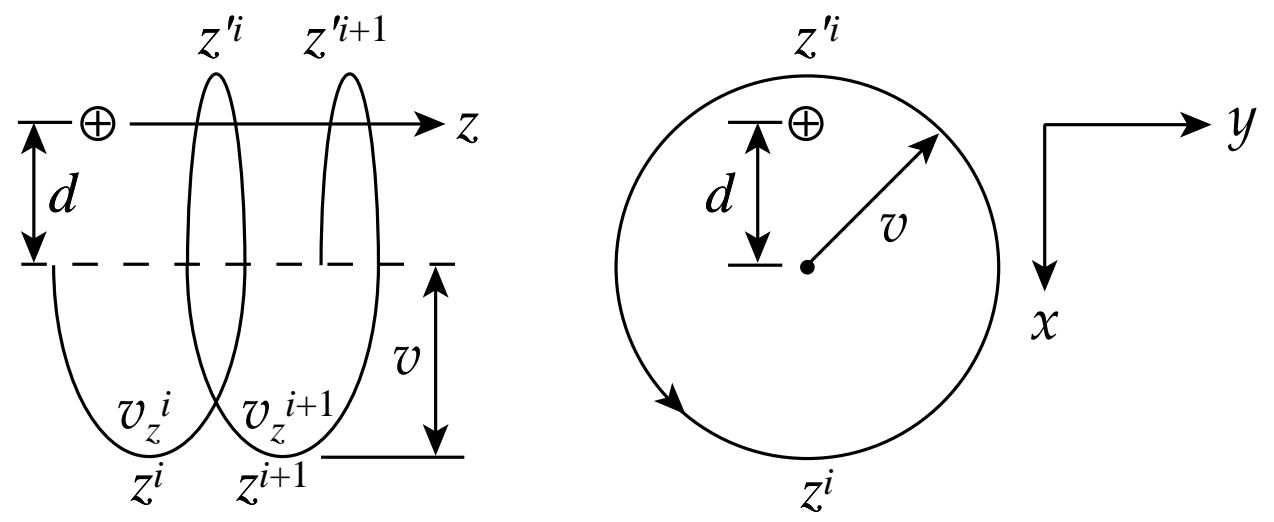

Figure 1.3: Variables in the map.

Under this consideration we now construct the map as follows. Let $d$ be the distance between the ion and the guiding center line. In Fig. 1.3 we illustrate a configuration for the construction of the map. In this figure $v_{z}^{i}$ and $z^{i}$ are defined as the $z$ and $v_{z}$ at the farthest points of the electron from the ion in the $x y$ plane, and $z^{\prime i}$ is defined as the $z$ at the closest points of the electron from the ion in the $x y$ plane for each cyclotron motion. First we construct an auxiliary map for the variable pair $\left(v_{z}^{i}, z^{i}\right)$. From the equation of motion the change in $v_{z}$ at the farthest point to the ion during one cyclotron motion is evaluated as

$$
v_{z}^{i+1}-v_{z}^{i} \approx \int_{0}^{2 \pi} \frac{z(t) d t}{\left[z(t)^{2}+v^{2}+d^{2}-2 v d \cos t\right]^{3 / 2}},
$$

where we have approximated that the cyclotron radius $\rho_{\mathrm{e}}(t) \approx v$ and $d(t) \approx d$ 
with given constants $v$ and $d$, and approximated that the electron does a uniform circular motion of period $2 \pi$. Moreover, because of the condition (1.11) of the small perturbation during each cyclotron period, we may approximate $z(t)$ by a constant $z^{i}$. This approximation is justified as follows. If $z \ll v$, the integrand in equation (1.12) is a sharp impulse peaked at $z^{\prime i}$, hence in the range of the main contribution to the integral $\delta z \ll z$. If $z \gtrsim v$, then $\delta z \ll z$ during one cyclotron period. Then we have

$$
\begin{aligned}
v_{z}^{i+1}-v_{z}^{i} & \approx \int_{0}^{2 \pi} \frac{z^{i} d t}{\left[{z^{\prime}}^{2}+v^{2}+d^{2}-2 v d \cos t\right]^{3 / 2}} \\
& =\frac{4 z^{\prime i} \sqrt{(d-v)^{2}+{z^{\prime}}^{2}}}{\left(d^{2}-v^{2}+z^{i^{2}}\right)^{2}+4 v^{2}{z^{\prime}}^{i^{2}}} E\left[\frac{-4 d v}{(d-v)^{2}+{z^{\prime}}^{i^{2}}}\right],
\end{aligned}
$$

where $E(m)$ is the complete elliptic integral of the second kind.

To estimate the change in $z^{\prime}$, we further approximate that the electron has a constant $v_{z} \approx v_{z}^{i+1}$ between $z^{i}$ and $z^{i+1}$. Then we have

$$
{z^{\prime}}^{i+1}-{z^{\prime}}^{i}=2 \pi v_{z}^{i+1} .
$$

A set of equations (1.13) and (1.14) gives a two dimensional area-preserving map. In this map the two variables $\left(v_{z}^{i}, z^{\prime i}\right)$ are defined at different points, i.e., the aphelion for $v_{z}^{i}$ and the perihelion for $z^{\prime}$, respectively.

In order to have a map with two variables defined at the same point, e.g., the aphelion, we use the pair $\left(v_{z}^{i}, z^{i}\right)$ and approximate as

$$
z^{i}={z^{\prime}}^{i}-\pi v_{z}^{i}
$$


Then we obtain a final form of the map for $\left(v_{z}^{i}, z^{i}\right)$, which is also area-preserving,

$$
\left\{\begin{aligned}
v_{z}^{i+1}=v_{z}^{i}+ & \frac{4\left(z^{i}+\pi v_{z}^{i}\right) \sqrt{(d-v)^{2}+\left(z^{i}+\pi v_{z}^{i}\right)^{2}}}{\left[d^{2}-v^{2}+\left(z^{i}+\pi v_{z}^{i}\right)^{2}\right]^{2}+4 v^{2}\left(z^{i}+\pi v_{z}^{i}\right)^{2}} \\
& \times E\left[\frac{-4 d v}{(d-v)^{2}+\left(z^{i}+\pi v_{z}^{i}\right)^{2}}\right] \\
z^{i+1}=z^{i}+ & \pi\left(v_{z}^{i}+v_{z}^{i+1}\right) .
\end{aligned}\right.
$$

We call this map the "magneto-Coulomb map".

The auxiliary map has a simple form, but is not symmetric in the sense that when the electron approaches the ion, $\left|z^{\prime i}\right|<\left|z^{i}\right|$, while the electron leaves the ion, $\left|z^{i}\right|>\left|z^{i}\right|$. This asymmetry will results in the asymmetry in the phase space plot. The "magneto-Coulomb map" gives a symmetric phase space plot, although it looks more complicated. Moreover, there is one degree of gauge freedom in the map, i.e., the coordinate $z^{i}$ can be evaluated at any point within the cyclotron period. The auxiliary map and the "magneto-Coulomb map" are just two choices of the gauge.

Previously, the effort to construct a map for the period in the $z$ direction was carried. It was not successful since the motion is chaotic, and during one period in the $z$ direction there exist too many cyclotron periods.

In order to have a criterion for the electron to escape without being bounced back in the $z$ direction, it is convenient to write the map in terms of the Keplerian energy $\varepsilon_{z}$ and its canonical conjugate $\tau$ defined by 


$$
\left\{\begin{aligned}
\varepsilon_{z} & =\frac{1}{2} v_{z}^{2}-\frac{1}{|z|} \\
\tau & =\operatorname{Sign}(z)\left(\frac{v_{z}}{\varepsilon_{z}\left(-2 \varepsilon_{z}+v_{z}^{2}\right)}-\frac{1}{\sqrt{2}\left|\varepsilon_{z}\right|^{3 / 2}} \tan ^{-1} \frac{v_{z}}{\sqrt{2\left|\varepsilon_{z}\right|}}\right) .
\end{aligned}\right.
$$

The derivation of $\tau$ will be presented at the end of this section. The comparison of the chaotic scattering with the case in which the electron moves in a straight line along the $z$ axis without the magnetic field with the same initial values $v_{z}^{0}$ and $z^{0}$ shows that, if the latter electron can escape, i.e., $\varepsilon_{z}^{0}=\frac{1}{2}\left(v_{z}^{0}\right)^{2}-\frac{1}{\left|z^{0}\right|}>0$, then the former one can also escape. Then the electron escape criterion is

$$
\varepsilon_{z} \geq 0 \quad \text { and } \quad z v_{z} \geq 0
$$

Here we have introduced the second condition for the sign of $z v_{z}$, to ensure that the electron is leaving the ion and will never come back. This criterion can be used in not only the map iterations, but also the numerical integrations. We know the electron will definitely escape if (1.18) is satisfied at some time. However, if (1.18) is not satisfied at some time, then the electron may or may not escape in the future. This uncertainty indicates the chaos.

The variable $\tau$ is calculated from the equation of motion for the onedimensional Kepler problem without the magnetic field,

$$
\frac{d v_{z}}{d \tau}=-\operatorname{Sign}(z) \frac{1}{z^{2}}
$$


and the relation $\varepsilon_{z}=\frac{1}{2} v_{z}^{2}-\frac{1}{|z|}$. We have

$$
\begin{aligned}
\tau & =-\operatorname{Sign}(z) \int^{v_{z}} d v_{z}\left(\frac{1}{2} v_{z}^{2}-\varepsilon_{z}\right)^{-2} \\
& =\operatorname{Sign}(z)\left(\frac{v_{z}}{\varepsilon_{z}\left(-2 \varepsilon_{z}+v_{z}^{2}\right)}-\frac{1}{\sqrt{2}\left|\varepsilon_{z}\right|^{3 / 2}} \tan ^{-1} \frac{v_{z}}{\sqrt{2\left|\varepsilon_{z}\right|}}\right)+C
\end{aligned}
$$

The integration constant $C=0$ is determined by imposing the condition that $\tau$ is continuous at $z=0$ where $\varepsilon_{z} \rightarrow-\infty$. In general an extra constant should be added to the right hand side of Eqn. (1.20) to make $\tau$ continuous, when $z$ changes sign. It happens in the neighbourhood of $z=0$ or $\varepsilon_{z}=-\infty$. The constant is, up to a sign,

$$
\left.\frac{\sqrt{2}}{\left|\varepsilon_{z}\right|^{3 / 2}} \tan ^{-1} \frac{v_{z}}{\sqrt{2\left|\varepsilon_{z}\right|}}\right|_{z=0^{-}} ^{z=0^{+}}=0,
$$

since $\varepsilon_{z} \rightarrow-\infty$ as $z \rightarrow 0$.

We note that Eq (1.20) ensures area-preserving,

$$
\frac{\partial\left(\varepsilon_{z}, \tau\right)}{\partial\left(v_{z}, z\right)}=1
$$

Moreover, Eqn. (1.22) has a homogeous solution for $\tau$, namely the time $\tau$ has a factor $g\left(\varepsilon_{z}\right)$ of gauge freedom, which can be any function of $\varepsilon_{z}\left(v_{z}, z\right)$.

\subsection{Comparison of map with numerical integration}

In order to see the accuracy of our map, we compare the results from the map to those from the numerical integration. Because of the choice of the coordinate system in Fig. 1.3, the initial condition $\left(z^{0}, v_{z}^{0}\right)$ and the parameters 

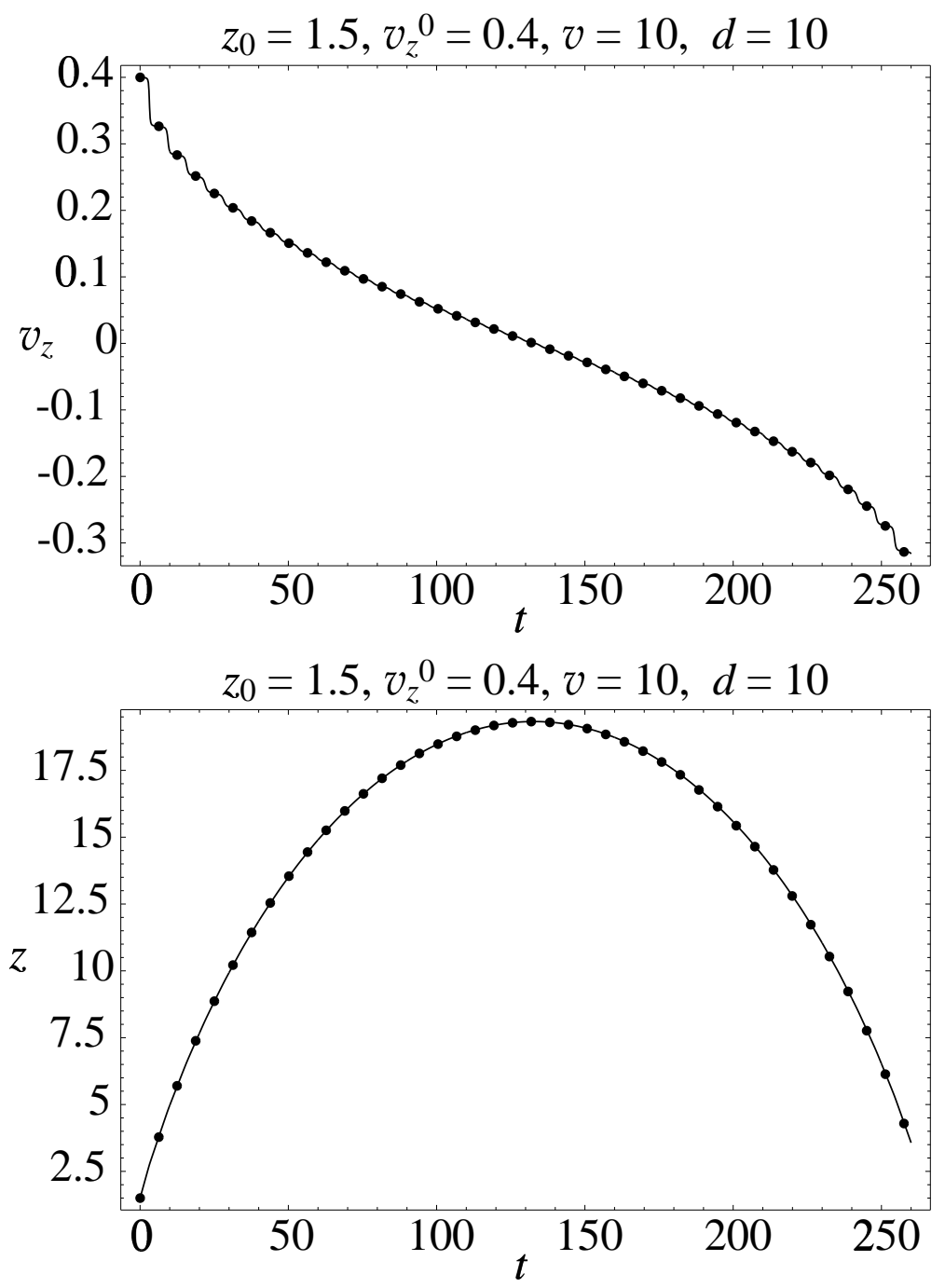

Figure 1.4: The comparsion between the map and the numerical integration for $\left(z, v_{z}\right)$ vs $t$. The initial condition is $z^{0}=1.5, v_{z}^{0}=0.4, v=10$ and $d=10$. The cyclotron period is $2 \pi$. 
$(v, d)$ in the map correspond to $x=v+d, y=0, v_{x}=0, v_{y}=v, z=z^{0}$ and $v_{z}=v_{z}^{0}$ in the numerical integration. In the numerical integration, $\left(z^{i+1}, v_{z}^{i+1}\right)$ are calculated at the next farest point to the ion in the $x y$ plane, and compared with the map. Big error from the map result may occur when the electron passes the ion in case that $v \sim d$ and $v \lesssim 1$. The numerical integration has been performed by Mathematica with Gauss-Kronrod method.

In Fig. 1.4 we show the comparison bewtween the map and the numerical integration for the time evolution of $\left\{z(t), v_{z}(t)\right\}$ with $z^{0}=1.5, v_{z}^{0}=0.4$, $v=10$ and $d=10$, where the lines denote the results from the numerical integration and the dots denote the results from the map. The agreement between the map and the numerical integration is excellent.

In Fig. 1.5 we show the comparison between the map and the numerical integration in the phase space $\left(\varepsilon_{z}, \tau\right)$ plot for the initial condition: $z^{0}=1.1$, $v_{z}^{0}=0, v=10$ and $d=9$. From the map (1.17), we see that $\varepsilon_{z} \rightarrow-\infty$ as $\tau \rightarrow 0$ (that corresponds to $z \rightarrow 0$ ). In Fig. 1.5, the points corresponding to large $\left|\varepsilon_{z}\right|$ are not shown.

For the individual trajectory there is a slight difference between the map and the numerical integration. However, the ensemble of the points has essentially the same structure (e.g., the location of the islands and the chaotic region), which is a well-known feature of the phase space plots in the chaotic dynamics that are generated from the different algorithms. 

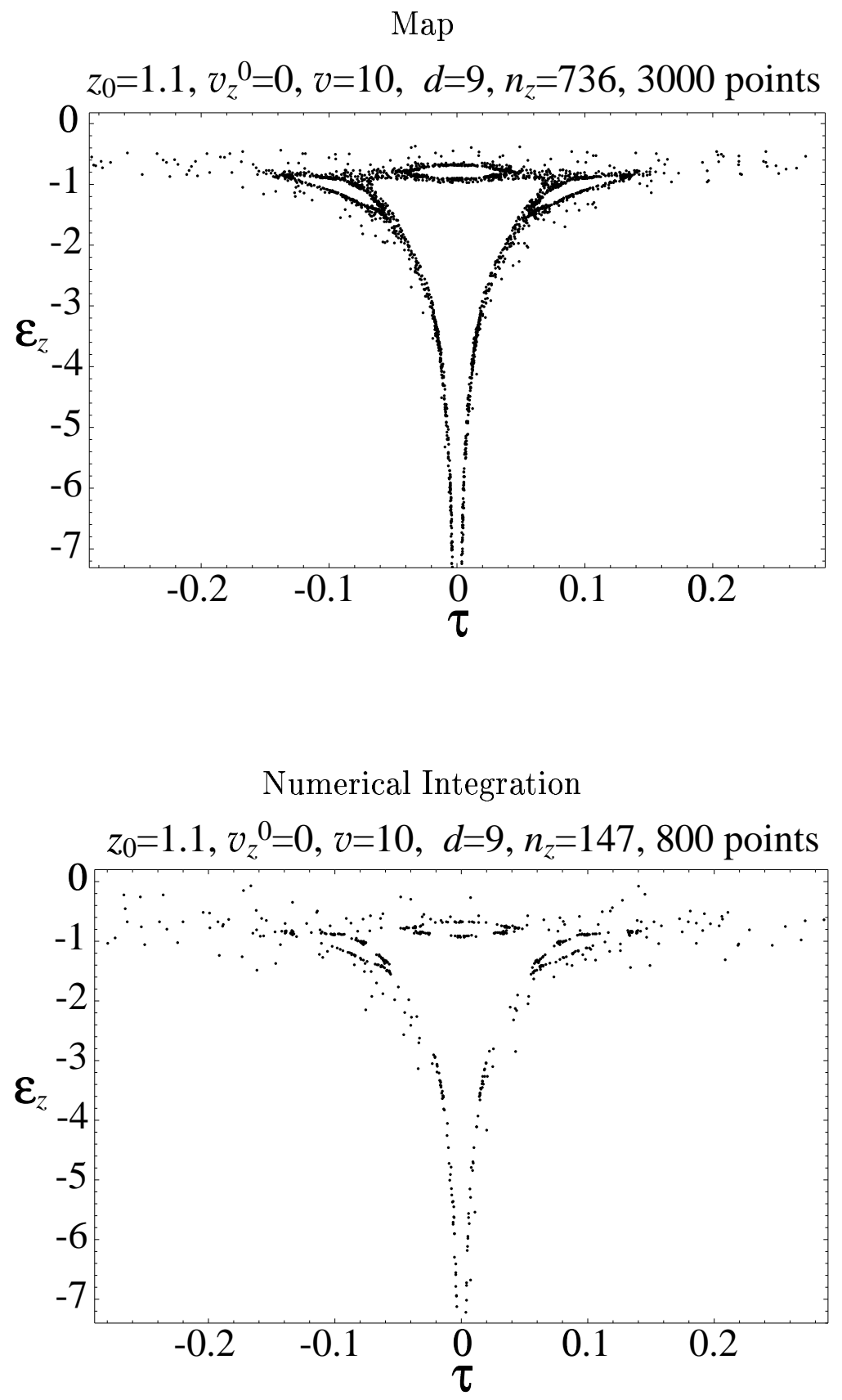

Figure 1.5: The comparison of the phase space $\left(\varepsilon_{z}, \tau\right)$ plots between the map and the numerical integration for the trapped chaotic motion near scattering regime. The initial condition is $z^{0}=1.1, v_{z}^{0}=0, v=10$ and $d=9$. 


\subsection{Phase space plots}

In this section, we shall show the phase space plots $\left(\varepsilon_{z}, \tau\right)$ that have been created from the map for several situations of the trapped motion and of the scattering motion.

In Fig. 1.6, we have plotted the trapped motion near the scattering regime. Fig. 1.6(a) has been created from four initial conditions: $z^{0}=$ $0.8,1.0,1.1$ and 1.5 with the same $v_{z}^{0}=0, v=10$ and $d=8.9$. For the chaotic motion, we have plotted up to 1000 iterations. Fig. 1.6(b) is also the trapped motion, but is much closer to the scattering regime than the ones in Fig. 1.6(a). The figure has been created from a single initial condition, $z^{0}=10, v_{z}^{0}=0$, $v=10$ and $d=8.6$. Note that the domain of $\tau$ is much larger and $\varepsilon_{z}$ is more closer to zero than the ones in Fig. 1.6(a).

In Fig. 1.7 we have plotted a scattering motion. The initial condition is $z^{0}=5, v_{z}^{0}=0, v=10$ and $d=10.8$. After 768 cyclotron periods, we have $\varepsilon_{z}>0$ and the electron has escaped toward the infinity from the ion. When the electron passes the ion each time, the point jumps randomly from one loop to another, and it eventually escapes. We notice the large size of the figure in $\tau$ direction, and smaller value of $\left|\varepsilon_{z}\right|$. Larger scale of $\tau$ indicates that there are more cyclotron periods between two bouncings, and the motion is more complicated. The scales of $\tau$ and $\varepsilon_{z}$ satisfy Keplerian relation approximately

$$
\left(\tau_{\max }-\tau_{\min }\right) \approx \frac{\pi}{2 \sqrt{2}}\left(-\varepsilon_{z \max }\right)^{-3 / 2}
$$


(a)

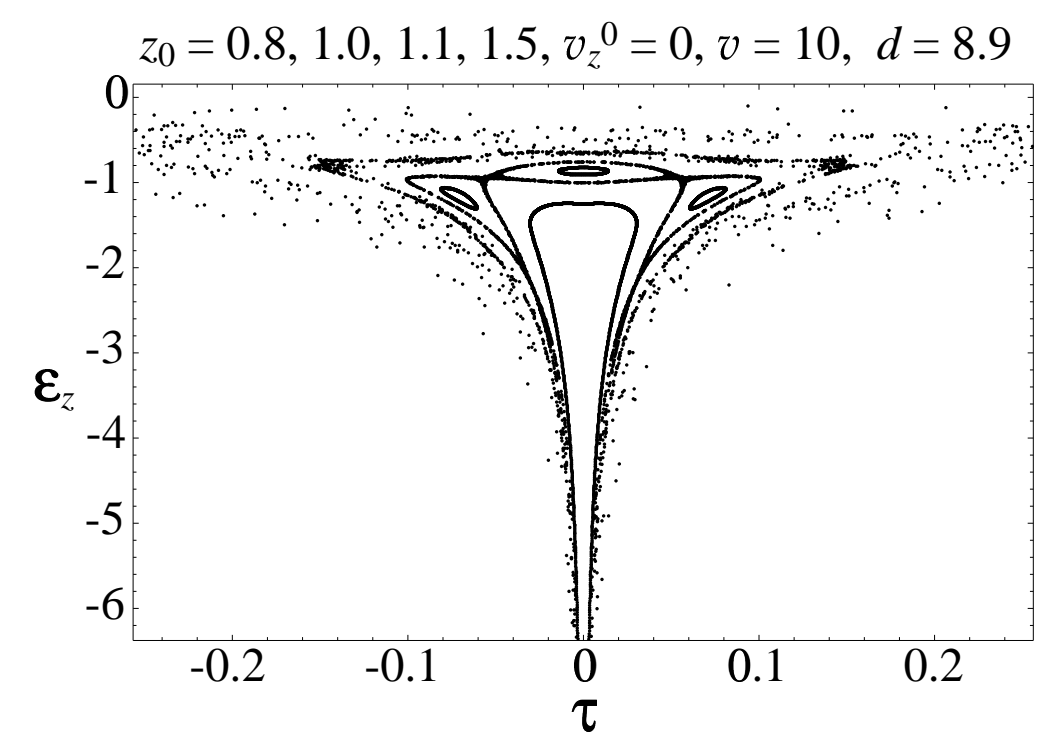

(b)

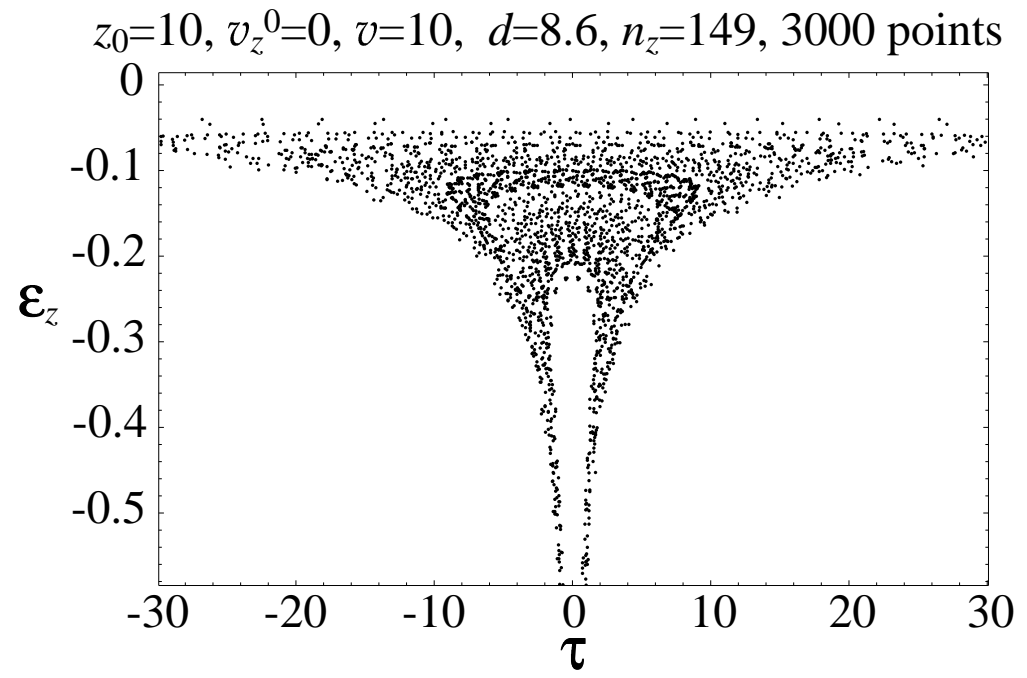

Figure 1.6: Trapped chaotic motion in $\left(\varepsilon_{z}, \tau\right)$ near scattering regime. 


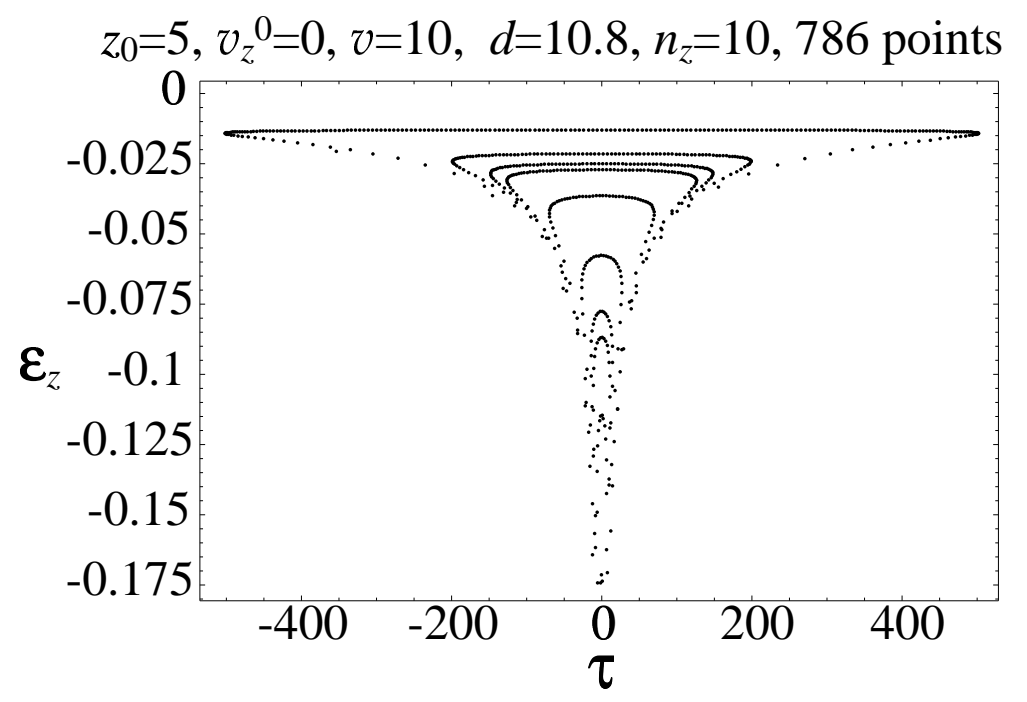

Figure 1.7: Phase space plot for scattering motion from map.

In Fig. 1.8 we show the islands of the trapped orbits inside the chaotic scattering region. In Fig. 1.8(a) the gray points are the scattered orbits generated from two initial conditions: $z^{0}=1.5$ and 1.7, with the same $v_{z}^{0}=0$, $v=10$ and $d=8.9$. These points have escaped to $\varepsilon_{z}>0$ after 7835 and 2091 cyclotron periods, respectively. The dark points in Fig. 1.8(a) are the periodical points of period 13, with the initial condition: $z^{0}=1.6, v_{z}^{0}=0$, $v=10$ and $d=8.9$. The magnified section of the right-most dark point is shown in Fig. 1.8(b). We see the island structure of the trapped motion. The points are generated from four different initial conditions: $z^{0}=$ $1.583,1.584,1.59$ and 1.595 , with the same $v_{z}^{0}=0, v=10$ and $d=8.9$. The case with $z^{0}=1.583$ gives the scattered orbit. 
(a)

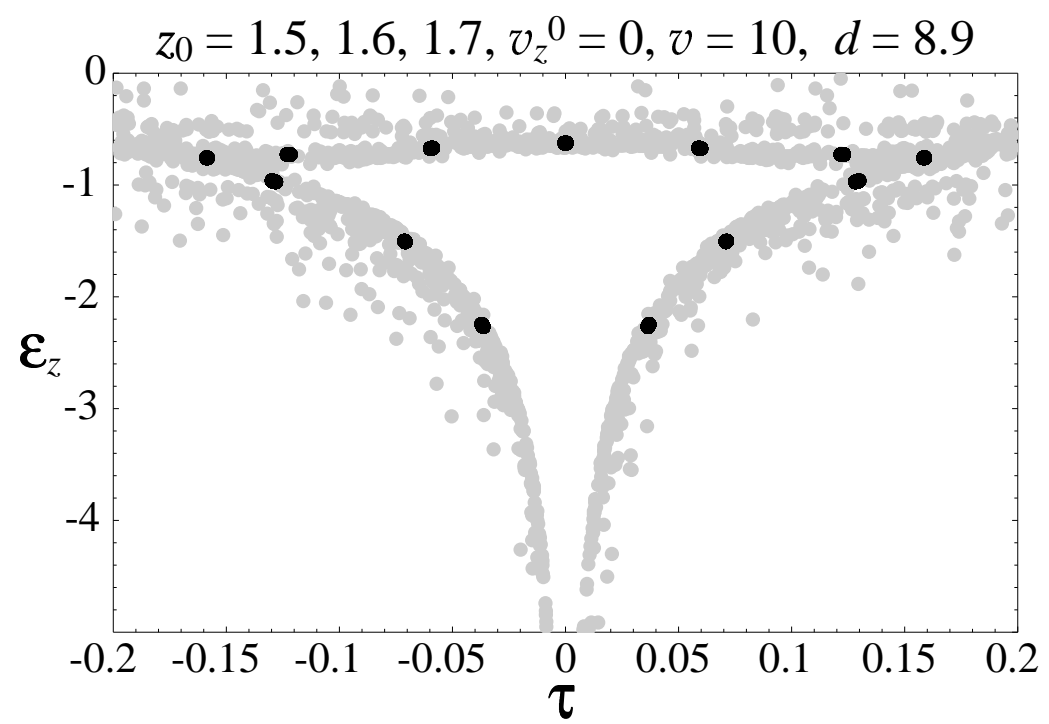

(b)

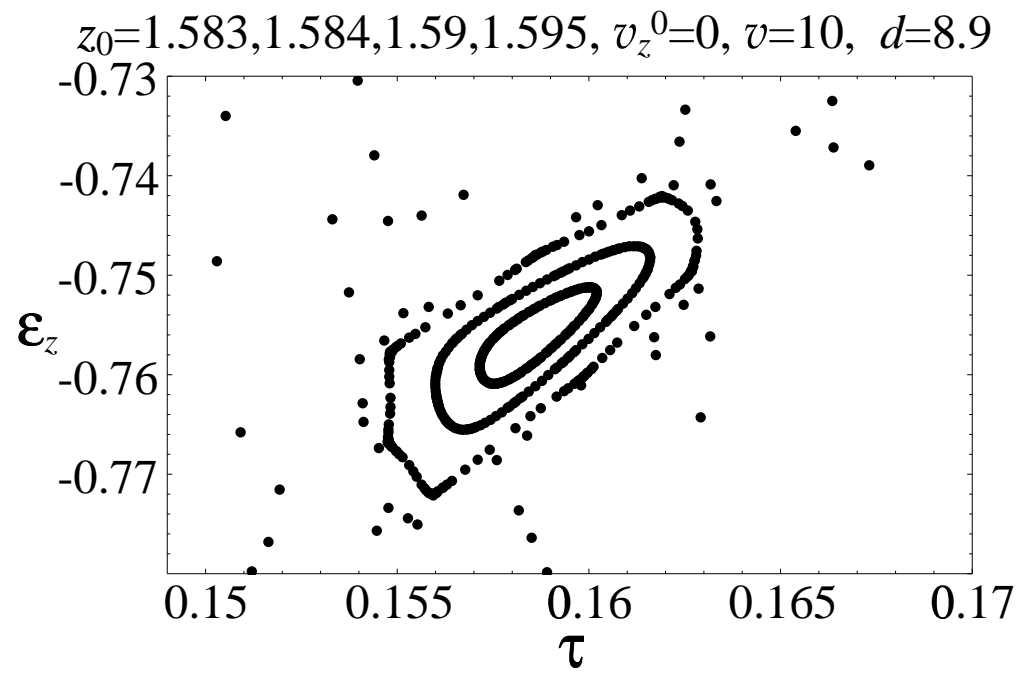

Figure 1.8: Islands of the trapped motion inside the scattering region. 


\subsection{Classification of motion}

In this section we consider a $d$-dependence of the motion of the electron for a given $v$ and with a given initial condition $\left(z^{0}, v_{z}^{0}\right)$. By increasing $d$, we found four different types of motion. They are

1. If $d \ll v$, the motion is trapped.

2. If $d \sim v$, the motion is chaotic scattering.

3. If $d \gg v$, the motion is trapped again.

4. If we increase $d$ further, we then have non-chaotic scattering motion.

In Fig. 1.9 we show the four corresponding orbits that are generated from the numerical integration. The initial condition $z^{0}=2.0, v_{z}^{0}=0.2$ and the parameter $v=10$ are the same for all the four cases, but the parameter $d$ is different, i.e., $d=8.7,9.4,11.3$ and 51 , respectively.

The existence of the four regions can be explained as follows. The equation of motion in the $z$ direction is

$$
\ddot{z}=-\frac{z}{\left(z^{2}+r_{\perp}^{2}\right)^{3 / 2}}
$$

where $r_{\perp}$ is the component of $\boldsymbol{r}$ perpendicular to the $z$ direction. If $d \ll v$, then $r_{\perp} \approx v$, and Eq (1.24) may be approximated as

$$
\ddot{z} \approx-\frac{z}{\left(z^{2}+v^{2}\right)^{3 / 2}}
$$


1 Trapping

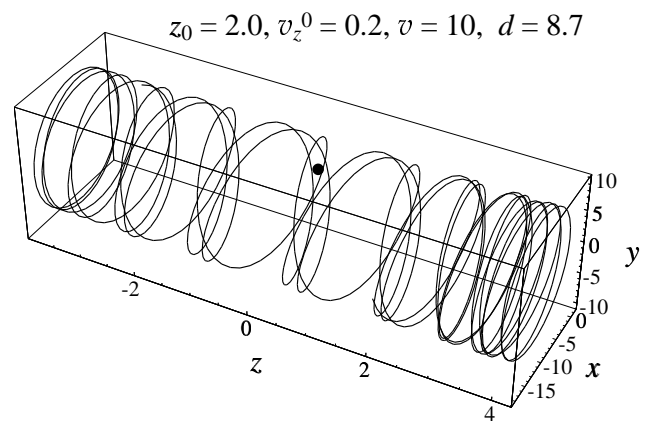

3 Trapping

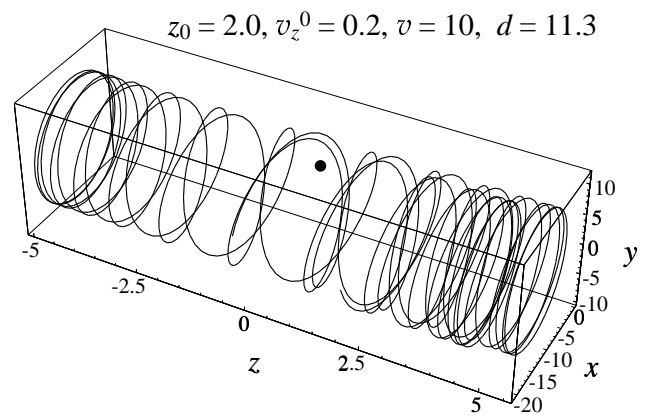

2 Chaotic scattering

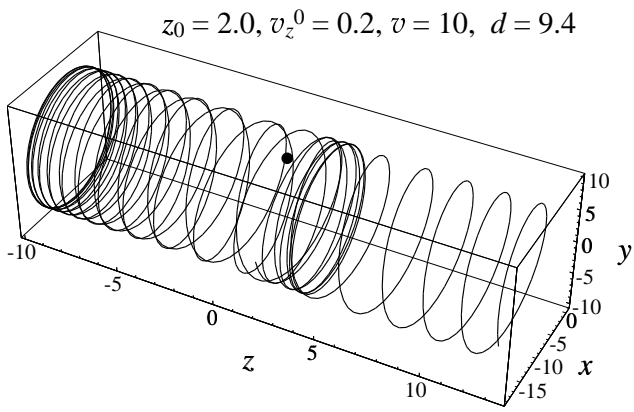

4 Regular scattering

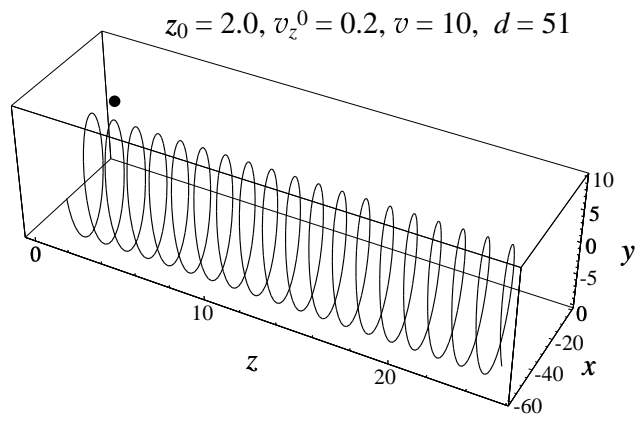

Figure 1.9: Four types of orbits, $v=10$.

On the other hand, if $d \gg v$, then $r_{\perp} \approx d$, and Eq (1.24) may be approximated as

$$
\ddot{z} \approx-\frac{z}{\left(z^{2}+d^{2}\right)^{3 / 2}} .
$$

In each of the above two cases, we see a well-defined potential in the $z$ direction from the equation of motion. If the total energy (i.e., the potential energy plus the kinetic energy) in the $z$ direction is negative, the electron is trapped. Then for a proper initial condition such that the total energy is negative in each case, we have two trapping regions, denoted by region 1 and region 3 in Fig. 1.10. 


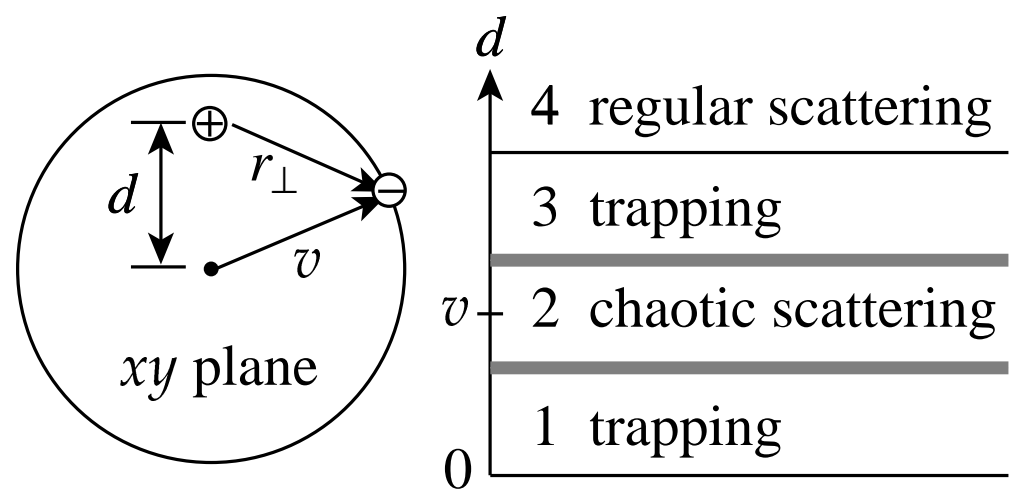

Figure 1.10: Four types of motion.

For each potential if we increase the energy to the positive region, then we obtain the regular scattering motion. Region 4 is a regular scattering region. For some initial conditions, region 1 may become a regular scattering region, and region 3 may disappear. The interaction for the potential (1.25) is relatively larger than the potential (1.26), and the potential is relatively welldefined in the latter case. As a result, the chaotic scattering region is much larger in the region 2 than in the region around the border between 3 and 4 . Indeed, there exists a chaotic scattering region between 3 and 4 . However, this region is so narrow that one cannot easily detect the chaotic scattering.

The map and the potential approximation may not be valid for small $v$. However, the same qualitative description and the classification of the orbits should hold in general. 


\section{7 $\quad$ Fractal structure}

It is well known that whenever we have a chaotic motion, there appears a fractal structure in some variables. In this section we show the fractal structure in the $v_{z}^{\mathrm{f}}$, i.e., the value of $v_{z}$ when the electron escapes to infinity, and the bouncing number $n_{z}$ of the electron, where $n_{z}$ is defined as the number of times $v_{z}$ changes its sign before the electron escapes.

In Fig. 1.11(a) we show $v_{z}^{\mathrm{f}}$ as a function of $v_{z}^{0}$, with $v_{z}^{0} \in[0.2,0.25]$, $z^{0}=1.5, v=10$ and $d=10$. It is seen that by slightly changing $v_{z}^{0}$, there are regions where $v_{z}^{\mathrm{f}}$ has a big change. This indicates the sensitivity in the initial condition. Moreover, there are smooth regions where $v_{z}^{\mathrm{f}}$ changes slowly. This shows that even inside the chaotic scattering regime, there exist regular regions.

In Fig. 1.11(b) we magnify a small section in $v_{z}^{0}$, i.e., $v_{z}^{0} \in[0.2,0.2001]$ to see the fractal structure. This figure shows that inside the chaotic scattering regime, there is self-similarity, which is typical of the fractal structure.

In Fig. 1.12(a), we show $n_{z}$ vs $v_{z}^{0}$ for the same domain of Fig. 1.11(a). It can be seen that whenever $n_{z}$ is constant, $v_{z}^{\mathrm{f}}$ is smooth, while in the chaotic region for $v_{z}^{\mathrm{f}}$, we have the chaotic region for $n_{z}$. We again magnify the same interval in $v_{z}^{0}$ for $n_{z}$, as shown in Fig. 1.12(b), and one can see again the fractal structure in $n_{z}$.

The fractal structure is also obtained from the numerical integration, as functions of $v$ and $b$. The comparison with the map shows agreement. 
(a)

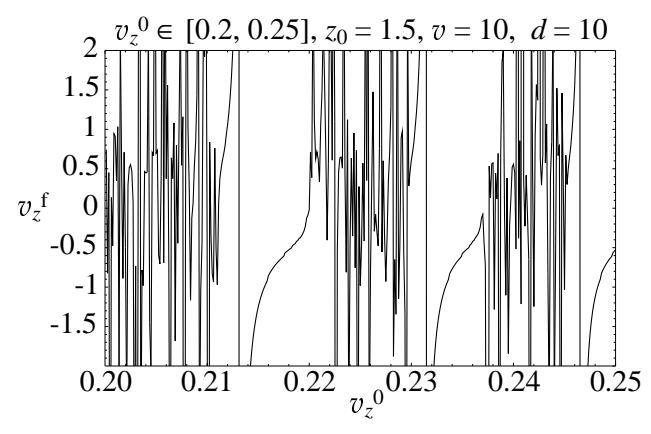

(b)

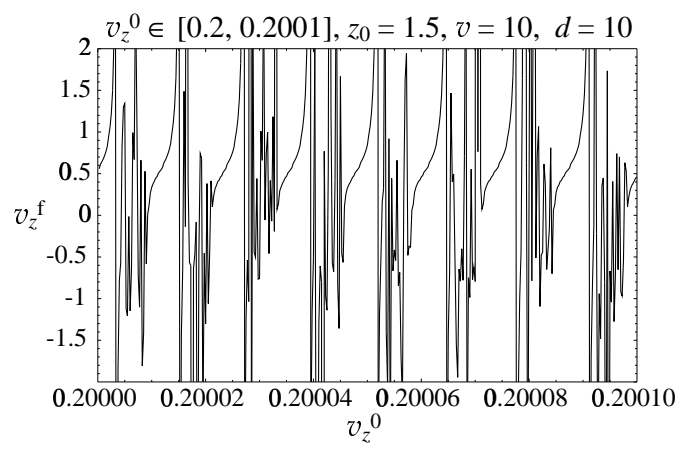

Figure 1.11: Fractal structure obtained from the map in $v_{z}^{\mathrm{f}}$ vs $v_{z}^{0}$ for initial conditions $v_{z}^{0} \in$ (a) $[0.2,0.25]$ and (b) $[0.2,0.2001], z^{0}=1.5, v=10$ and $d=10$.

(a)

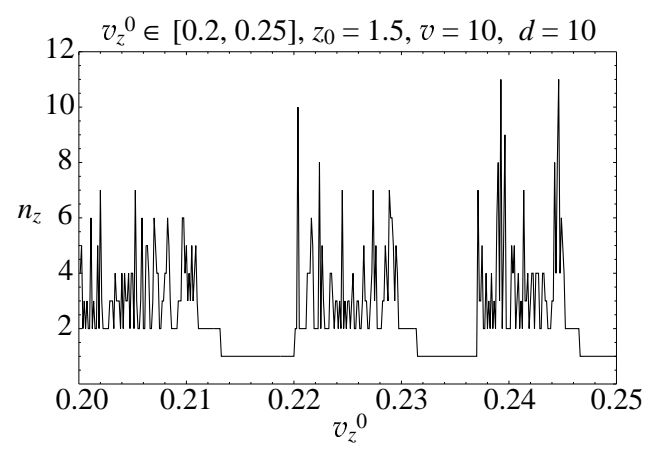

(b)

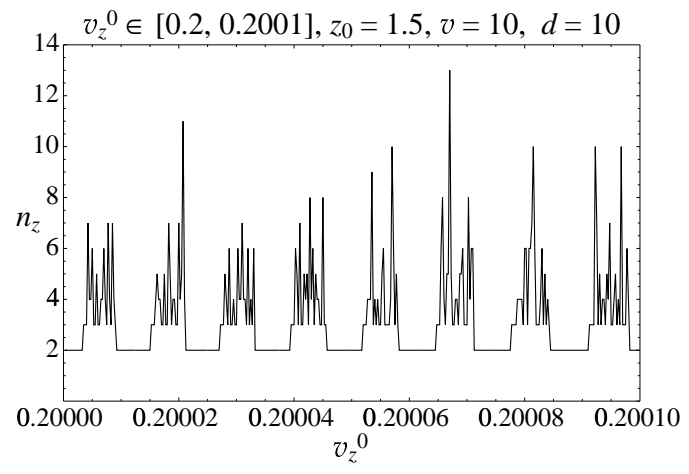

Figure 1.12: Fractal structure obtained from the map in $n_{z}$ vs $v_{z}^{0}$ for initial conditions $v_{z}^{0} \in$ (a) $[0.2,0.25]$ and (b) $[0.2,0.2001], z^{0}=1.5, v=10$ and $d=10$. 


\subsection{Width of chaotic scattering region}

In this section we estimate the size of the chaotic scattering region. For the case that $v$ is small, the effect of the Coulomb force becomes a large perturbation over the cyclotron motion. This suggest that smaller the velocity, wider the chaotic region. In this section we shall verify this prediction and numerically estimate the width of the chaotic region in the impact parameter as a function of $v$. However, our map is restricted only for the large values of $v$. For this reason, we perform the numerical integration of the equation of motion to find the width, instead of using our map.

We take the initial condition of the electron to be parallel to $z$ with a velocity $v$. In this case, the initial condition is specified by the impact parameter $b$ and the velocity $v$, and we use the numerical integration to compute the width $\delta b$ of the chaotic region for fixed $v$. Since the chaotic scattering region is much lager in region 2 in Fig. 1.10, we restrict our interest in this domain.

In order to compute $\delta b$, we need to define the upper bound and the lower bound of the chaotic region. The bouncing number is an indication of the chaos. To this end we first note that if $b$ is too large, there is no bouncing motion of the electron, i.e., $n_{z}=0$. For this case we have a regular scattering. Hence, the upper bound of the chaotic motion is determined by the boundary in $b$ between $n_{z}=0$ and $n_{z} \neq 0$. On the other hand, if $b$ is too small, there is only one bouncing, i.e., $n_{z}=1$, which corresponds to the other regular scattering. Hence, the lower bound of the chaotic motion is determined by the boundary in $b$ for small $b$ region between $n_{z}=1$ and $n_{z} \neq 1$. 


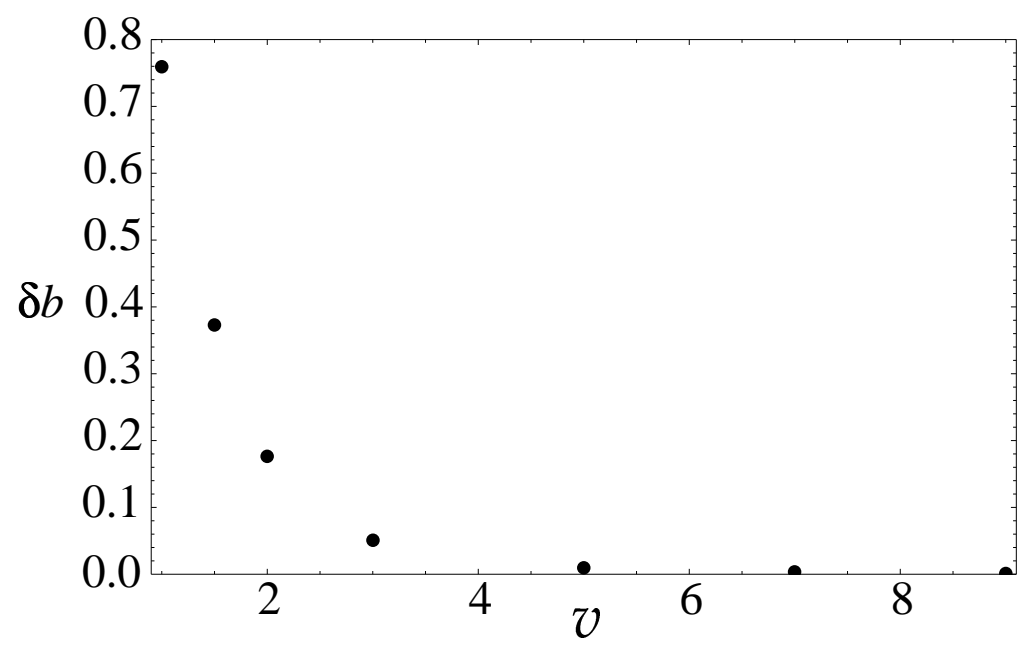

Figure 1.13: Width $\delta b$ of chaotic scattering region in the impact parameter $b$ as a function of velocity $v$.

In Fig. 1.13 we show $\delta b$ as a function of $v$ from the results of the numerical integration. We see that $v$ decreases as $\delta b$ increases. The chaotic scattering is more likely to be observed for small $v$.

\subsection{Radiation energy loss}

Finally we give a comment on the effect of the radiation damping. During the cyclotron motion, the electron loses energy because of the radiation. In this paper, we have neglected this effect. Here we estimate the radiation energy loss and show that the loss is very small. Let us consider the dimensionless

power $P(t)$ radiated by an electron undergoing a dimensionless acceleration 
$a(t)$. It is given by

$$
P(t)=\frac{\omega_{\mathrm{c}}}{m_{\mathrm{e}}} \frac{e^{2}}{6 \pi \epsilon_{0} c^{3}} a^{2}(t)=\frac{e^{3}}{6 \pi \epsilon_{0} c^{3} m_{\mathrm{e}}^{2}} B a^{2}(t)=\left[1.1 \times 10^{-12} \mathrm{~T}^{-1}\right] B a^{2}(t),
$$

and the radiated energy is

$$
\delta \mathcal{E}=\int d t P(t)=\left[1.1 \times 10^{-12} \mathrm{~T}^{-1}\right] B \int d t a^{2}(t) .
$$

In the case of $v \sim 1$, we have $a(t) \sim 1$. Then the incident electron has an

energy $\mathcal{E}=\frac{1}{2} v^{2} \sim 1$. We can see from Eq (1.28) that, in a time period of thousands cyclotron periods or less the energy radiated from an electron can be neglected, compared to the electron energy $\mathcal{E}$.

\subsection{Anomalous transport}

Suppose this scattering occurs in the plasma enviroment, then one can expect that plasma transport may become anomalous under the influence of the chaotic scattering. Our results suggest that the smaller $v$, the more anomalous the plasma transport. In the following we present several plasma enviroments, where $v \lesssim 1$. These cases are the ultracold neutral plasma [11], the white dwarf atmosphere, the neutron star atmosphere, with $v=1.0,2.4$ and 0.5 , respectively. We expect the chaotic scattering has a stronger influence on these plasma enviroments. With the relation $\frac{3}{2} k_{\mathrm{B}} T_{\mathrm{e}}=$ $\frac{1}{2} m_{\mathrm{e}}\left(v \omega_{\mathrm{c}} r_{0}\right)^{2}$, where $T_{\mathrm{e}}$ is the electron temperature, the dimensionless velocity can be expressed as

$$
v=\left(3 k_{\mathrm{B}}\right)^{1 / 2}\left(4 \pi \epsilon_{0} m_{\mathrm{e}}^{2}\right)^{1 / 3} e^{-1} \frac{T_{\mathrm{e}}^{1 / 2}}{(Z B)^{1 / 3}}=\left[0.18 \mathrm{~K}^{-1 / 2} \mathrm{~T}^{1 / 3}\right] \frac{T_{\mathrm{e}}^{1 / 2}}{(Z B)^{1 / 3}},
$$


Table 1.1: Representative plasmas where chaotic scattering is predicted.

\begin{tabular}{c|c|c|c}
\hline \hline $\begin{array}{c}\text { Plasma } \\
\text { enviroments }\end{array}$ & $\begin{array}{c}\text { Ultracold } \\
\text { neutral plasma }\end{array}$ & $\begin{array}{c}\text { White dwarf } \\
\text { atmosphere }\end{array}$ & $\begin{array}{c}\text { Neutron star } \\
\text { atmosphere }\end{array}$ \\
\hline$T_{\mathrm{e}}(\mathrm{K})$ & 0.1 & $5.0 \times 10^{4}$ & $1.0 \times 10^{6}$ \\
$B(\mathrm{~T})$ & $4.0 \times 10^{-4}$ & $1.0 \times 10^{4}$ & $1.0 \times 10^{8}$ \\
$v$ & 1.0 & 2.4 & 0.5 \\
$n_{\mathrm{e}}\left(\mathrm{m}^{-3}\right)$ & $2.0 \times 10^{15}$ & $1.0 \times 10^{22}$ & $1.0 \times 10^{30}$ \\
$n_{\mathrm{e}}^{-1 / 3}(\mathrm{~m})$ & $7.9 \times 10^{-6}$ & $4.6 \times 10^{-8}$ & $1.0 \times 10^{-10}$ \\
$r_{0}(\mathrm{~m})$ & $3.7 \times 10^{-5}$ & $4.3 \times 10^{-10}$ & $9.2 \times 10^{-13}$ \\
$\delta b(\mathrm{~m})$ & $2.8 \times 10^{-5}$ & $1.0 \times 10^{-9}$ & $1.3 \times 10^{-12}$ \\
$b_{90}(\mathrm{~m})$ & $5.6 \times 10^{-5}$ & $1.1 \times 10^{-10}$ & $5.6 \times 10^{-12}$ \\
$\rho_{\mathrm{e}}(\mathrm{m})$ & $3.0 \times 10^{-5}$ & $8.6 \times 10^{-10}$ & $3.8 \times 10^{-13}$ \\
$\lambda_{\mathrm{De}}(\mathrm{m})$ & $4.9 \times 10^{-7}$ & $1.5 \times 10^{-7}$ & $6.9 \times 10^{-11}$ \\
$\lambda_{\mathrm{e}}(\mathrm{m})$ & $2.4 \times 10^{-7}$ & $3.3 \times 10^{-10}$ & $7.5 \times 10^{-11}$ \\
\hline \hline
\end{tabular}

where and $\mathrm{K}$ and $\mathrm{T}$ denote Kelvin and Tesla. Table 1.1 lists some relative parameters for these three plasma enviroments. The parameters $b_{90}, \lambda_{\mathrm{e}}$ and $\lambda_{\text {De }}$ are the impact parameter for $90^{\circ}$ Rutherford scattering, thermal DeBroglie wavelength and Debye length in the dimensional units respectively,

$$
\begin{gathered}
b_{90}=\frac{Z e^{2}}{12 \pi \epsilon_{0} k_{\mathrm{B}} T_{\mathrm{e}}}, \\
\lambda_{\mathrm{e}}=\frac{2 \pi \hbar}{\sqrt{2 \pi m_{\mathrm{e}} k_{\mathrm{B}} T_{\mathrm{e}}}},
\end{gathered}
$$




$$
\lambda_{\mathrm{De}}=\sqrt{\frac{\epsilon_{0} k_{\mathrm{B}} T_{\mathrm{e}}}{n_{\mathrm{e}} e^{2}}} .
$$

From (1.29), it can be seen that increasing the ion charge is the same as increasing the magnetic field. In fact, some experiments are done with a charged sphere as the ion in the plasma enviroment [12].

Generalizations of the magneto-Coulomb map which could be applied to the study of the anomalous transport is discussed in the Appendix.

For fusion plasmas with a typical temperature of $10^{7} \mathrm{Kelvin}$ and a typical magnetic field of 10 Tesla, the dimensionless velocity $v \gg 1$. Therefore the electric force and the magnetic force are weakly coupled: during the scattering period, the influence of the magnetic field is negligible; before and after the scattering process, the electric field is negligible. Thus this chaotic scattering has little influence for $v \gg 1$, and the Rutherford scattering is a very accurate model to calculate the resistivity for the ohmic heating in tokamak plasmas. However, if the ion is replaced with a charged sphere, the relation $v \lesssim 1$ may be satisfied. This experiment has been done by J. Godino et al. [12]. 


\section{Chapter 2}

\section{Reversed Magnetic Shear Confinement for Ignitor}

\subsection{Introduction to tokamaks}

The highest rate of fusion reactions takes place in the deuterium-tritium (D-T) mixture. The equation for the $\mathrm{D}-\mathrm{T}$ reaction is

$$
\mathrm{D}+\mathrm{T} \rightarrow \mathrm{He}^{4}(3.52 \mathrm{MeV})+\mathrm{n}(14.06 \mathrm{MeV})
$$

The optimal energy for the $\mathrm{D}-\mathrm{T}$ reaction is of the order of $10-100 \mathrm{keV}$. The most promising method of supplying the energy is to heat the D-T mixture to a sufficiently high temperature.

In order to have a self-sustaining burning plasma, the power generated by the fusion reaction should at least compensate for the power loss of the plasma. For a steady state plasma, power balance gives

$$
P_{\mathrm{H}}=P_{\mathrm{L}},
$$

where $P_{\mathrm{H}}$ is the heating power and $P_{\mathrm{L}}$ is the power loss. The critical condition for a self-sustaining burning plasma is that the alpha heating power $P_{\alpha}$ itself has to compensate for the power loss $P_{\mathrm{L}}$, which gives the ignition condition,

$$
P_{\alpha}=P_{\mathrm{L}}
$$


The concept of tokamak comes from the idea of magnetic thermal insulation: a high-temperature plasma immersed in a strong magnetic field should have a strongly decreased thermal conductivity across the magnetic field due to the fact that the trajectories of charged particles look like compact helical lines wound on the magnetic field lines. Thus magnetic field lines should be closed in such a way that a magnetic flux tube would have a toriodal form.

Ohmic heating is the simplest method of plasma heating. During the ohmic heating process, a current is generated along the toroidal direction of the tokamak by an induced electric field. The resistance is caused by electron-ion collision. The ohmic heating rate is calculated by

$$
P_{\mathrm{oh}}=j E
$$

where $j$ and $E$ are the current and the electric field. The non-uniformity of the field causes the so-called neoclassical resistivity

$$
\eta_{\mathrm{n}}=\frac{\eta_{\mathrm{s}}}{\left(1-\epsilon^{1 / 2}\right)^{2}}
$$

where $\epsilon=r / R$ is the inverse of the aspect ratio and $\eta_{\mathrm{s}}$ is the Spitzer resistivity. One of the model used to calculate the plasma resistivity is the Spitzer resistivity

$$
\eta_{\mathrm{s}} \approx 3 \times 10^{8} Z_{\mathrm{eff}} T_{\mathrm{e}}^{-3 / 2} \Omega \cdot \mathrm{m}
$$

where $T_{\mathrm{e}}$ is the electron temperature in $\mathrm{keV}$ and $Z_{\text {eff }}$ is the effective ion charge number. The important feature of the plasma resistivity is that it drops significantly when the electron temperature goes up. Plasma with high impurity 
has a higher value of the effective ion charge $Z_{\text {eff }}$, which leads to a higher ohmic heating rate. However, higher level of impurity also leads to higher radiation power loss.

Since the inductive ohmic heating is of a pulsed type, it has its own drawbacks, e.g., small resistivities at high temperatures and interruption of the power output. There are other non-inductive current heating methods, e.g., neutral beam injection, lower hybrid current drive, fast wave electron current drive, fast wave minority ion current drive and ion-cyclotron current drive. They belong to two main categories, (1) the injection of enegetic neutral beams and (2) the resonant absorption of radio frequency electromagnetic waves.

The power loss of tokamak plasmas is mainly caused by transport. The minimal level of transport fluxes is determined by Coulomb collisions. This is called classical transport. In a tokamak the situation becomes more complicated due to drift of particles in a non-uniform magnetic field. This kind of transport is called neoclassical transport, and is usually much higher than the classical one. However, the main contribution to the transport comes from the so-called anomalous transport, which is from turbulent diffusion caused by fluctuations in plasma. In the turbulence-induced transport, free energy released by instabilities drives a steady level of fluctuations in the associated perturbed quantities, and results in a transport of particles and energy. These fluctuations may be either electrostatic or electromagnetic and appear as the saturated states of microinstabilities.

Experiments show that tokamaks are subjected to a variety of macroin- 
stabilities which can mainly be attributed to identifiable magnetohydrodynamic modes. The major advantage of the tokamak configuration is the possibility of stabilizing magnetohydrodynamic instabilities by a strong toroidal magnetic field. Let $\phi$ and $\theta$ be the poloidal and the toroidal angles of the tokamak torus. For a large aspect-ratio $(R / a)$ tokamak the modes take the form $\exp i(m \theta-n \phi)$, with $m$ and $n$ being the poloidal and the toroidal mode numbers. One important variable, the safety factor, is defined as

$$
q=\frac{B_{\phi} r}{B_{\theta} R}
$$

where $R$ is the major radius and $r$ is the radial coordinate for the tokamak, and $B_{\phi}, B_{\theta}$ being the toroidal and the poloidal magnetic fields. $q$ has a lot to do with the plasma instabilities. For a magnetohydrodynamic mode with mode numbers $m$ and $n$, the safety factor is $q=m / n$. The most dangerous modes are $q=1 / 1,2 / 1,3 / 2$.

In a stable phase of the tokamak discharge, the central temperature and density experience periodical oscillations of relaxation type. Each period consists of slow temperature rise with subsequent fast drop. During the ramping phase heating raises the temperature and at the collapse the thermal energy is released to the outer part of the plasma through a heat pulse. This is called sawtooth oscillation, which belongs to the disruptive type of instabilities. The oscillation precusors are observed to be $m=1, n=1$ mode, which is associated with the existence of $q=1$ surface. 


\subsection{Introduction to Ignitor}

Ignitor $[13,14]$ is a proposed high-magnetic-field, high-density, compact tokamak machine. The original idea of Ignitor was derived from the Alcator machines at MIT, where high tokamak performances were observed on highdensity and peaked density discharges. The main goal of Ignitor is to achieve

low temperature $(T(0) \lesssim 15 \mathrm{keV})$ D-T ignition, where the alpha heating power does not exceed twice of the ohmic heating power. Ignitor is designed to be able to achieve ignition with pure ohmic heating. Since the resistivity decreases with temperature, with pure ohmic heating, low temperature ignition is desired. For $15 \mathrm{keV}$, Lawson criterion derived from (2.3) requires $n(0) \tau_{E} \gtrsim$ $4 \times 10^{20} \mathrm{~m}^{-3} \cdot \mathrm{s}$. Thus high density is also required. The ignition point is defined as the regime where the fusion alpha heating compensates for the thermal energy losses due to anomalous transport and radiation. The high plasma toroidal current results in a strong ohmic heating rate. The average current density $j \sim 1 \mathrm{kA} \cdot \mathrm{cm}^{-3}$, and the poloidal field $B_{\theta} \approx 3.9 \mathrm{~T}$. High poloidal field also confines most of the alpha particles in order for their energy to be deposited into the plasma. Although the Ignitor machine is expected to achieve ignition condition with only ohmic heating, the ion-cyclotron radio frequency heating can broaden the operating scenarios. Ignition is achieved through transient states rather than steady states.

There are several other proposed tokamak machines currently, e.g., ITER-FEAT, FIRE. The comparison of Ignitor with ITER-FEAT and FIRE is shown in terms of some parameters in Table 2.1 . 
Table 2.1: Ignitor compared with some other proposed tokamak machines

\begin{tabular}{cccc}
\hline name & Ignitor & FIRE & ITER-TEAT \\
\hline major radius $R(\mathrm{~m})$ & 1.32 & 2.0 & 6.2 \\
\hline minor radius $a(\mathrm{~m})$ & 0.47 & 0.525 & 2.0 \\
\hline toroidal field $B_{\phi}(\mathrm{T})$ & 13 & 10 & 5.3 \\
\hline plasma current $I_{\mathrm{p}}(\mathrm{MA})$ & 12 & 7.7 & 15 \\
\hline
\end{tabular}

\subsection{Problems and motivation}

Some critical issues for Ignitor are the production of peaked density profiles on a time scale of several energy confinement times, the control of the current penetration for the optimization of ohmic heating and sawtooth aviodance [15].

Several high performance tokamak operating regimes have been achieved in experiments through the peaking of density profiles. These include the improved ohmic confinement regime produced in ASDEX [16], the pellet enhanced performance mode in Alcator-C and JET, and the supershot mode in TFTR. In these tokamak regimes, the peaked core density profiles bring down the $\eta_{\mathrm{i}}\left(=d \ln T_{\mathrm{i}} / d \ln n_{\mathrm{i}}\right)$ value below the critical threshold for ion temperature gradient mode, and help to produce internal transport barriers by generating the large shear radial electric field, which is required for turbulence suppression, through the deep density gradient. In addition to enhancing the confinement, peaked density profiles are also necessary for optimizing the fusion reaction rate and alpha heating power of a tokamak plasma, and when combined with centrally peaked temperature profiles, would help to reach the 
ignition condition early. It is thus desirable to seek and analyze the density profile control schemes that effectively lead to density profile peaking in transport simulations of burning plasma experiments on machines such as Ignitor.

Previous simulations of Ignitor experiments either simply utilized density profiles with fixed peaking shape [14] or obtained flat density profiles when the self-consistent evolution of which was allowed [15]. Efforts have been made to increase core plasma density by pellet injection in Ignitor simulation, however, the density profile peaking seems to only last for a very short time period. Here we demonstrate in transport simulation the scheme of producing peaked density profiles through fast current ramping, which eventually leads to the ignition of Ignitor plasma when sawtooth event is avoided. The basic mechanism is that plasma current ramping with sufficient rate and proper timing generates a non-monotonic $q$ profile with reversed magnetic shear during the evolution of magnetic flux surface. The magnetic shear $s$ is defined as

$$
s=\frac{r}{q} \frac{d q}{d r} .
$$

An internal transport barrier forms in the reversed shear region which, combined with the properly programmed tokamak edge gas puffing, produces a centrally peaked plasma density profile in a timely manner. Since the reversed magnetic shear is a natural by-product of rapid plasma current ramping as well as a well confirmed mechanism in the formation of transport barrier, such a scheme of density profile peaking is intrinsic to the ohmic heating process as in Ignitor, and independent of the particular transport model used in simulations. The creation of the internal barrier due to the reversed magnetic shear 
has been observed in several tokamaks [17-21] and confirmed by numerical simulations $[22,23]$.

Current ramping to achieve reversed shear confinement enhancement and peaked density profiles are crucial in achieving ignition conditions in Ignitor. Since Ignitor is a high-field, high-current tokamak, it is possible to use a fast plasma current ramping to produce reversed magnetic shear for a fairly long time period.

Here the transport model JETTO is used as a standard model in simulations to demonstrate the scheme. JETTO is an empirical model with a mixed Bohm and gyro-Bohm scaling. It has been bench-marked in various confinement regimes with experimental data from several different tokamak machines [24]. Even though, it is still uncertain whether or not the transport scaling in JETTO could be applied to the compact tokamak regime with high magnetic field, high density and high current. However, the issue of extrapolation of transport models should not digress our purpose here in an essential way, due to the universal nature of the current ramping scheme. In this chapter enhancement confinement is explored, and a general scheme leading to density profile peaking is shown using the transport model JETTO in the BALDUR simulations. In these simulations, peaked density profiles result from the formation of internal transport barrier due to reversed magnetic shear, which is produced by controlled plasma current and volume-averaged density ramping. Such a programmed Ohmic heating scheme is demonstrated to be an effective approach to the ignition of a burning D-T plasma [25]. Reversed magnetic 
shear plasma confinement has become one of the main approaches to achieving fusion grade plasmas in tokamaks.

\subsection{Tokamak plasma transport code BALDUR}

BALDUR is a one and one half dimensional transport code to simulate a wide range of tokamak plasmas [26,27]. The transport equations solved are one dimensional (radial dimension) flux surface averaged (poloidal averaged) equations. BALDUR contains integrated modules for models computing the sources (neutral beam injection, fusion, and auxiliary heating), sinks (radiative, scrape-off layer loss), fluxes (neoclassical and anomalous, particle and heat, of electron, main and impurity ions), equilibrium shapes (magnetohydrodynamic equilibrium), large scale instabilities (sawtooth), and boundary conditions. Specially it computes the transport evolution of the density and temperature for electron and up to six ion species, as well as the evolution of the poloidal magnetic field. Having a baseline or default transport model in, BALDUR can also interface with various other transport models, with itself as a test bed.

The BALDUR code solves the diffusion equations for particles, energies and poloidal magnetic field. The diffusion equation for poloidal magnetic field is discussed in the next section. The diffusion equations for the particles and energies in the BALDUR code are

$$
\frac{\partial n_{a}}{\partial t}=-\frac{1}{r} \frac{\partial}{\partial r}\left(r \Gamma_{a}\right)+S_{a}
$$




$$
\frac{\partial E_{j}}{\partial t}=-\frac{1}{r} \frac{\partial}{\partial r}\left(r q_{j}\right)+Q_{j}
$$

where $n_{a}$ denotes the particle density for species $a, E_{j}$ denotes the energy density for electrons or ions. $\Gamma_{a}$ and $q_{j}$ denote the particle fluxes and the energy fluxes. $S_{a}$ denote the particle sources from ionization, recombination, nuclear reactions, edge gas puffing and recycling. $Q_{j}$ denote denote energy sources from ionization, recombination, charge exchange, radiation, heating, nuclear reactions, edge gas puffing and recycling.

The inner boundary conditions on the diffusion equations are

$$
\left.\frac{\partial n_{a}}{\partial t}\right|_{r=0}=\left.\frac{\partial E_{j}}{\partial t}\right|_{r=0}=\left.\frac{\partial B_{\theta}}{\partial t}\right|_{r=0}=0,
$$

where $B_{\theta}$ is the poloidal magnetic field introduced in the next section. At the outer boundary, the values or the radial gradients of the variables are fixed.

\subsection{Ohmic heating and current penetration}

One important process during the current ramping is the time evolution for the plasma toroidal current or the poloidal field along the radial direction of the tokamak torus. The poloidal field diffusion equation

$$
\frac{\partial B_{\theta}}{\partial t}=\frac{1}{\mu_{0}} \frac{\partial}{\partial r}\left(\frac{\eta}{r} \frac{\partial\left(r B_{\theta}\right)}{\partial r}\right)-\frac{4 \pi}{\mu_{0} c} \frac{\partial}{\partial r}\left(\eta j_{\mathrm{b}}\right)
$$

comes from the combination of the two of the Maxwell's equations,

$$
\left\{\begin{aligned}
\nabla \times \boldsymbol{E} & =-\frac{\partial \boldsymbol{B}}{\partial t} \\
\nabla \times \boldsymbol{B} & =\mu_{0} \boldsymbol{j}+\frac{1}{c^{2}} \frac{\partial \boldsymbol{E}}{\partial t}
\end{aligned}\right.
$$


where the last term in the second equation of (2.13) is dropped since it is too small. Note that in order to get (2.12), the relation between $j$ and $E$ from Ohm's law,

$$
E=\eta\left(j-j_{b}\right)
$$

is needed. $j$ is the total plasma toroidal current and $j-j_{b}$ is the current component driven by the electric field $E . j_{\mathrm{b}}$ is the current component which is not driven by the electric field. $j_{\mathrm{b}}$ includes terms for toroidal effects on ion orbits, pitch-angle scattering of ions and electrons into trapped orbits and the neoclassical electron return current. Then the ohmic heating power density is

$$
P_{\mathrm{oh}}=j E=\eta j\left(j-j_{b}\right) .
$$

The resistivity $\eta$ has a neoclassical definition,

$$
\eta=\frac{\left(2 \pi m_{\mathrm{e}}\right)^{1 / 2} e^{2} \lambda_{\mathrm{e}}}{0.75 T_{\mathrm{e}}^{3 / 2} \Lambda_{\mathrm{E}} f_{\text {Trap }}}
$$

The columb logarithm is

$$
\lambda_{\mathrm{e}}=\ln \left(\frac{\lambda_{\mathrm{De}}}{b_{\mathrm{min}}}\right)=\ln \left[1.09 \times 10^{11} T_{\mathrm{e}} n_{\mathrm{e}}^{1 / 2} Z_{\mathrm{eff}}\right],
$$

where the closest distance of approach

$$
b_{\min }=\frac{e^{2}}{4 \pi \epsilon_{0} k_{\mathrm{B}} T_{\mathrm{e}}},
$$

and Debye length is

$$
\lambda_{\mathrm{De}}=\left(\frac{\epsilon_{0} T_{\mathrm{e}}}{n e^{2}}\right)^{1 / 2}
$$




$$
\Lambda_{\mathrm{E}}^{-1}=\frac{Z_{\mathrm{eff}}\left(2.67+Z_{\mathrm{eff}}\right)}{3.40\left(1.13+Z_{\mathrm{eff}}\right)}
$$

The trapped electron factor is

$$
f_{\text {Trap }}=\left(1-f_{\mathrm{T} \xi}\right)\left(1-f_{\mathrm{T} \xi} C_{\mathrm{R}}\right)
$$

where

$$
f_{\mathrm{T} \xi}=\frac{f_{\mathrm{T}}}{1+\xi \nu_{\mathrm{e}}^{*}}
$$

and

$$
f_{\mathrm{T}}=1-\frac{(1-\epsilon)^{2}}{\left(1-\epsilon^{2}\right)^{1 / 2}\left(1+1.46 \epsilon^{1 / 2}\right)} .
$$

$\epsilon=a / R$ is the aspect ratio.

$$
\begin{gathered}
C_{\mathrm{R}}=\frac{0.56\left(3-Z_{\mathrm{eff}}\right)}{Z_{\mathrm{eff}}\left(3+Z_{\mathrm{eff}}\right)} \\
\xi=0.58+0.2 Z_{\mathrm{eff}}
\end{gathered}
$$

The ratio of the effective collision frequency for trapped particles to their bounce frequency (Wesson 4.14) is

$$
\nu_{\mathrm{e}}^{*}=\frac{(2 \pi)^{1 / 2}}{0.75} \frac{B_{\mathrm{T}}}{B_{\theta}} \frac{n_{\mathrm{e}} e^{4} \lambda_{\mathrm{e}}}{T_{\mathrm{e}}^{2}} \frac{R^{3 / 2}}{r^{1 / 2}} .
$$

Neglect the beam current $J_{\mathrm{b}}$ and let $\eta$ be constant, (2.12) becomes

$$
\frac{\partial B_{\theta}}{\partial t}=\frac{\eta}{\mu_{0}} \frac{\partial}{\partial r}\left(\frac{1}{r} \frac{\partial\left(r B_{\theta}\right)}{\partial r}\right) .
$$


For steady state, we have uniform current distribution,

$$
B_{\theta}=\frac{B_{\theta}(r=a)}{a} r
$$

For non-steady state,

$$
B_{\theta}=\frac{B_{\theta}(r=a)}{a} r+\sum_{i=1}^{\infty} c_{i} \exp \left[-\frac{\eta}{\mu_{0}}\left(\frac{\mu_{i}}{a}\right)^{2} t\right] J_{1}\left(\frac{\mu_{i}}{a} r\right)
$$

where $\mu_{i}$ is the $i$-th zero of Bessel function $J_{1}$. The time scale of current diffusion can be approximated by

$$
\tau_{\mathrm{cd}}=\frac{\mu_{0}}{\eta}\left(\frac{a}{\mu_{1}}\right)^{2}
$$

where

$$
\mu_{1}=3.83171
$$

Sometimes, the constant factor $\mu_{1}^{2}$ is dropped, and

$$
\tau_{\mathrm{cd}} \approx \frac{\mu_{0} a^{2}}{\eta}
$$

An important feature of the ohmic heating is that when the temperature goes up, the resistivity goes down. For a centrally peaked temperature profile, as the current pinches in, the diffusion process becomes slower and slower, and the $q$ profile looks like being frozen in the core area.

\subsection{Tokamak circuit}

Consider the transformer flux produced in the central solenoid $\phi_{T}$ due to the $N_{T}$ windings of a current $I_{T}$ around an area $A_{T}$ and height $H_{T}$. Thus, 
the self-inductance of the central solenoid is approximately

$$
L_{T}=\frac{N_{T}^{2} \mu_{0} A_{T}}{H_{T}}
$$

There is a flux through $A_{T}$ due to the poloidal field from the plasma current of $M_{12} I_{p}$ and this flux opposes the flux from $I_{T}$. Thus, the transformer flux is

$$
\phi_{T}=L_{T} I_{T}-M_{12} I_{p}=N_{T} \phi_{T \text { per turn }}
$$

The back emf on the transform circuit is

$$
\mathcal{E}_{T}=-\frac{d \phi_{T}}{d t}=-L_{T} \frac{d I_{T}}{d t}+M_{12} \frac{d I_{p}}{d t}
$$

The poloidal field at the center of the tokamak torus is approximately

$$
B_{p}=\frac{\mu_{0} I_{p}}{2 \pi R}
$$

The mutual inductance is approximately

$$
M=\frac{\mu_{0} N_{T} A_{t}}{2 \pi R}
$$

The power provided to the transformer must be

$$
P_{T}=I_{T} V_{T}=I_{T}\left(R_{T} I_{T}+L_{T} \frac{d I_{T}}{d t}-M_{12} \frac{d I_{p}}{d t}\right)
$$

Now the self-inductance of the plasma current if flows on as a skin current at radius $r=a$ is

$$
L_{p}^{\mathrm{skin}}=\mu_{0} R_{0} \ln \left(\frac{R_{0}}{a}\right) .
$$


If the current has a profile

$$
j_{\phi}=j_{0}\left(1-\frac{r^{2}}{a^{2}}\right)^{\alpha_{j}} .
$$

Then one computes the poloidal field $B_{\theta}(r)$ and the stored energy and defined $\ell_{i}$ by

$$
W_{B}^{p}=\int_{r \leq a} \frac{B_{\theta}^{2}}{2 \mu_{0}} d^{3} x=\mu_{0} R \ell_{i} \frac{I_{p}^{2}}{2} .
$$

Thus the total energy stored in the magnetic field is

$$
W_{B}=\frac{1}{2} L_{p} I_{p}^{2}
$$

with

$$
L_{p}=\mu_{0} R_{0}\left[\ell n\left(\frac{R_{0}}{a}\right)+\ell_{i}\right] .
$$

For a uniform current profile given by $\alpha_{j}=0$ and $j_{0}=I / \pi a^{2}$ the value of $\ell_{i}=1 / 2$. Peaked profiles have $\ell_{i}$ around 1 to 1.5. The internal inductance $\ell_{i}$ is inferred from the $L_{p}$ and loop voltage inferred from the Lp measured by magnetic sensors for $B_{\mathrm{p}}$ and coils for the induced electric field just inside the vacuum chamber. The value of li is an important characterization of the shape of the current profile and is used to judge the approach to the MHD stability limit during the plasma discharge. and used to characterize the shape of the current profiles.

The flux of transformer field through the loop at major radius $R$ is

$$
\phi_{p}=L_{p} I_{p}-M_{21} I_{T}
$$

where an estimate of $M_{21}$ is 


$$
M_{21}=\frac{N \mu_{0} A_{T}}{H_{T}}
$$

using area $A_{T}$ and $B_{T}=\mu_{0} N_{T} I_{T} / H_{T}$ for the field in the air-core transformer. If the core is iron the $B_{T}=\mu_{F e} N_{T} I_{T} / H_{T}$. The back emf produced at radius $R_{0}$ is then

$$
\mathcal{E}_{p}=-\frac{d \phi_{p}}{d t}=-L_{p} \frac{d I_{p}}{d t}+M_{21} \frac{d I_{T}}{d t}
$$

From energy conservation we have that $M_{21}=M_{12}$ and $L_{p} L_{T}>M_{12}^{2}$.

For Tore Supra the transformer flux is about $15 \mathrm{~Wb}=15 \mathrm{~V} \cdot \mathrm{s}$ for example.

Now the transformer steps down the voltage and up the current by $N_{T}$ which can be 20 to 100 . Thus, the transfer of power from $T$ top is given by

$$
\frac{\mathcal{E}_{T}}{P}=N_{T} M_{1,2} \text { per turn } \frac{d I_{p}}{d t}=N_{T} \mathcal{E}_{p}
$$

\subsection{Alpha heating}

The D-T fusion reaction formula is

$$
\mathrm{D}+\mathrm{T} \rightarrow{ }^{4} \mathrm{He}(3.52 \mathrm{MeV})+\mathrm{n}(14.06 \mathrm{MeV})
$$

The $\mathrm{D}-\mathrm{T}$ fusion reaction rate is given by

$$
n_{\mathrm{D}} n_{\mathrm{T}}\langle\sigma v\rangle_{\mathrm{DT}}
$$


where $\langle\sigma v\rangle_{\mathrm{DT}}$ is the reaction rate. In the BALDUR code, the empirical formula for $\langle\sigma v\rangle_{\mathrm{DT}}$ is

$$
\begin{aligned}
\langle\sigma v\rangle_{\mathrm{DT}}= & \exp \left(-22.712 T_{\mathrm{i}}^{-0.275}-23.836\right. \\
& -9.393 \times 10^{-2} T_{\mathrm{i}}+7.994 \times 10^{-4} T_{\mathrm{i}}^{2} \\
& \left.-3.144 \times 10^{-6} T_{\mathrm{i}}^{3}\right)
\end{aligned}
$$

where $T_{\mathrm{i}}$ is in $\mathrm{keV}$ and $\langle\sigma v\rangle_{\mathrm{DT}}$ is in $\mathrm{m}^{3} \cdot \mathrm{s}^{-1}$.

Other empirical formula often used for $\langle\sigma v\rangle_{\mathrm{DT}}$ are

$$
\langle\sigma v\rangle_{\mathrm{DT}}=1.1 \times 10^{-22}\left(\frac{T_{\mathrm{i}}}{10}\right)^{s},
$$

where

$$
s= \begin{cases}3 & T_{\mathrm{i}} \leq 10 \\ 2 & T_{\mathrm{i}}>10\end{cases}
$$

and

$$
\langle\sigma v\rangle_{\mathrm{DT}}=3.7 \times 10^{-18} h\left(T_{\mathrm{i}}\right) T_{\mathrm{i}}^{-2 / 3} \exp \left(-\frac{20}{T_{\mathrm{i}}}\right)
$$

where

$$
h(x)=\left(1+\left(\frac{x}{70}\right)^{1.3}\right)^{-1}
$$

The alpha heating gives rise to the plasma energy as well as the particle sink and source terms. 


\subsection{Thermonuclear gain parameter}

The thermonuclear gain parameter is defined by

$$
Q=\frac{P_{\text {fus }}}{P_{\text {in }}}
$$

where $P_{\text {fus }}$ is the fusion power $\left(P_{\text {fus }}=5 P_{\alpha}\right.$ for a D-T reacting plasma), and the input power $P_{\text {in }}=P_{\text {oh }}+P_{\text {aux }}$. The total power balance equation is

$$
\frac{d W}{d t}=P_{\mathrm{oh}}+P_{\alpha}+P_{\mathrm{aux}}-P_{\mathrm{L}}
$$

where $W$ is the total energy of the plasma. In the ignited state $P_{\alpha}=P_{\mathrm{L}}$, so one may introduce the generalized gain parameter $Q_{*}$ defined by

$$
Q_{*}=\frac{P_{\text {fus }}}{P_{\mathrm{L}}-P_{\alpha}}=\frac{P_{\text {fus }}}{P_{\text {in }}-d W / d t}
$$

One can see that $Q_{*}$ becomes equal to $Q$ when $d W / d t=0$, i.e. $Q_{*}$ is the natural extension of the thermonuclear gain parameter under non-steady-state operation.

\subsection{Edge gas puffing and recycling}

The general discussion on the recycling of neutral atoms can be found in Chapter 10 of Goldston [28] and Chapter 9 of Wesson [29]. At the edge of the plasma, the electrons and the ions that escape from the plasma recombine into neutral atoms when they hit the containing vessel. These recycled neutrals then go back to the plasma, together with neutrals from edge gas puffing. In the boundary layer of the plasma, the neutrals interact with the electrons 
and the ions, thus two processes, e.g., ionization and charge exchange occur. Recombination in the plasma is usually negligible.

Because of the ionization due to electron-neutral interaction, the neutrals with velocity $v_{\mathrm{n}}$ can only penetrate a certain distance

$$
\lambda_{\mathrm{n}}=\frac{v_{\mathrm{n}}}{n_{\mathrm{e}}\left\langle\sigma_{\mathrm{ion}} v_{\mathrm{e}}\right\rangle}
$$

In the case of Ignitor, for $2 \mathrm{keV}$ electron temperature, the ionization rate is about $10^{-14} \mathrm{~m}^{3} \cdot \mathrm{s}^{-1}[29]$. At the edge of the Ignitor plasma, the electron density is about $2 \times 10^{20} \mathrm{~m}^{-3}$, the penetration length $\lambda_{\mathrm{n}}$ for $2 \mathrm{keV}$ neutrals is about $0.3 \mathrm{~m}$, which is smaller the minor radius of Ignitor. In the core region, the electrons have a much higher temperature and density, and the actual penetration length for the neutrals is much smaller than the minor radius of Ignitor.

In the simulation with the BALDUR code, the neutral density $n_{\mathrm{n}}$ at the core region is maintained at approximately $10^{12} \mathrm{~m}^{-3}$, three orders of magnitude lower than that at the edge of the plasma. However, with a high ionization rate $\left\langle\sigma_{\text {ion }} v_{\mathrm{e}}\right\rangle$, which is about $10^{-14} \mathrm{~m}^{3} \mathrm{~s}^{-1}$ in the temperature range $10-10^{4} \mathrm{eV}$, the source term from the ionization process is about $n_{\mathrm{n}} n_{\mathrm{e}}\left\langle\sigma_{\mathrm{ion}} v_{\mathrm{e}}\right\rangle \sim 10^{19} \mathrm{~m}^{-3} \mathrm{~s}^{-1}$, large enough to significantly raise the electron and ion density in the core region of the plasma.

The other atomic process is charge exchange, i.e., an energetic plasma hydrogen ion captures the electron from a lower-energy neutral. This process is particularly important for hydrogen, because the rate is greater than that 
for the ionization. The penetration of neutrals is largely influenced by charge exchange. There is not much energy exchanged by the charge-exchange collision, the emerging neutral has nearly the same energy as the incident plasma ion. For an plasma temperature of $10-100 \mathrm{keV}$, this cross section $\sigma_{\mathrm{cx}}$ is approximately $4 \times 10^{-19} \mathrm{~m}^{2}$, which is nearly two orders of magnitude larger than the ionization cross section. For a plasma with $T_{\mathrm{e}} \approx T_{\mathrm{i}}$, the charge-exchange rate is usually two to three times larger than the ionization rate [28]. The charge-exchange cross section is large because it is a resonant effect, where the initial and the final quantum states almost have no energy difference. For 2 $\mathrm{keV}$ ion temperature in the Ignitor simulation case, the charge-exchange rate $\left\langle\sigma_{\mathrm{cx}} v_{\mathrm{i}}\right\rangle$ is about $10^{-13} \mathrm{~m}^{3} \cdot \mathrm{s}^{-1}$. When low-energy neutrals are injected into the edge of a plasma, the charge exchange would increase the penetration of neutrals into hot dense plasmas by producing a series of generations of more energetic neutrals with energies comparable to the ions.

In the BALDUR code, the neutral gas source terms are computed using a Monte Carlo algorithm. The computation involves computing the trajectories of a specified number of sample particles for the neutral atoms, and then using these trajectories to calculate the source rates to be used by the energy and the particle balance equations. There are three types of neutral sources for each hydrogen species, edge source due to the recycling of the plasma ions and neutrals, edge source due to gas puffing, volume source due to charge exchange between injected beam neutrals and plasma ions and due to radiative recombination of plasma ions and electrons. 
In the simulation, the kinetic energy of the recycled hydrogenic neutrals is assumed to be equal to the ion temperature at the edge of the simulation. The rationale for this is that the edge neutrals undergo a succession of charge exchange events before they get to the edge of the simulation. This prescription works very well for TFTR supershot simulations [30].

Moreover, the edge gas puffing neutrals are also assumed to have an energy equal to the ion temperature at the edge of the simulation.

\subsection{Transport model JETTO and simulation scheme}

Here we have adopted the transport model JETTO in our simulations of Ignitor. JETTO is an empirical model containing several extensions to the Taroni-Bohm electron thermal transport model originally developed for JET L-mode experiments [31]. Erba et al. [32] first extended the TaroniBohm model to ion thermal transport and to the ohmic regime. By adding edge temperature gradient dependence, Erba et al. [33] further extended their model to H-mode regimes with transient and nonlocal effects. Later a gyroBohm transport term was included to account for experimental results on other tokamak devices with various sizes [24]. In the final model of JETTO, the thermal diffusivities of electrons and ions take the form [34]

$$
\begin{aligned}
\chi_{\mathrm{e}, \mathrm{i}} & =\chi_{\mathrm{e}, \mathrm{i}}^{\mathrm{B}}+\chi_{\mathrm{e}, \mathrm{i}}^{\mathrm{gB}} \quad \text { Bohm term }+ \text { gyro-Bohm term }, \\
\chi_{e, i}^{\mathrm{B}} & =\alpha_{\mathrm{e}, \mathrm{i}}^{\mathrm{B}} \frac{c T_{\mathrm{e}}}{e B} \frac{q^{2} a}{L_{p \mathrm{e}}}\left\langle L_{T_{\mathrm{e}}}^{*}\right\rangle_{\Delta V}^{-1} \quad \text { Bohm term }, \\
\chi_{\mathrm{e}, \mathrm{i}}^{\mathrm{gB}} & =\alpha_{\mathrm{e}, \mathrm{i}}^{\mathrm{gB}} \frac{c T_{\mathrm{e}}}{e B} \frac{\rho_{\mathrm{si}}}{L_{T \mathrm{e}}} \quad \text { gyro-Bohm term },
\end{aligned}
$$


where

$$
\left\langle L_{T_{\mathrm{e}}}^{*}\right\rangle_{\Delta V}^{-1}=\left|\left(T_{\mathrm{e}}(x=0.8)-T_{\mathrm{e}}(x=1)\right) / T_{\mathrm{e}}(x=1)\right|
$$

is the correction factor for nonlocality at the edge,

$$
L_{p \mathrm{e}}=\left|\frac{d \ln p_{\mathrm{e}}}{d r}\right|^{-1}
$$

and

$$
L_{T \mathrm{e}}=\left|\frac{d \ln T_{\mathrm{e}}}{d r}\right|^{-1}
$$

are the electron pressure and electron temperature gradient scale length, respectively, $\rho_{\mathrm{si}}$ is ion Larmor radius at the electron temperature, and the adopted values of the empirical transport coefficients are

$$
\begin{gathered}
\alpha_{\mathrm{Be}}=8 \times 10^{-5}, \quad \alpha_{\mathrm{Bi}}=2 \alpha_{\mathrm{Be}} \\
\alpha_{\mathrm{gBe}}=3.5 \times 10^{-2}, \quad \alpha_{\mathrm{gBi}}=\alpha_{\mathrm{gBe}} / 2
\end{gathered}
$$

as bench-marked from data in ITER and Tore Supra databases. The particle diffusivity of main gas ion species is modeled as

$$
D_{\mathrm{i}}=\left[c_{1}+\left(c_{2}-c_{1}\right) \frac{r}{a}\right] \frac{\chi_{\mathrm{e}} \chi_{\mathrm{i}}}{\chi_{\mathrm{e}}+\chi_{\mathrm{i}}}
$$

where $c_{1}=1$ and $c_{2}=0.3$ are empirical coefficients and $r / a$ is the normalized minor radius of device.

The Hydrogen ion particle flux $\Gamma^{i}$ and heat fluxes $q_{e, i}$ are calculated by

$$
\begin{aligned}
\Gamma^{i} & =-D_{i} \frac{\partial n_{i}}{\partial r} \\
q_{e, i} & =-n_{i, e} \chi_{i, e} \frac{\partial T_{i, e}}{\partial r}
\end{aligned}
$$


Weak and reversed magnetic shear has been shown to suppress several dominant magnetohydrodynamic and microinstabilities in tokamak, particularly those driven by unfavorable geodestic magnetic curvature [17, 18, 35-37]. Such suppression leads to the reduced turbulent transport accross the reversed shear region, resulting in the formation of internal transport barrier. In tokamak experiments with transport barriers, reversed shear works either alone or together with some other turbulence suppression mechanisms, such as the $\boldsymbol{E} \times \boldsymbol{B}$ flow shear. The mechanism of reversed shear is incorporated into the JETTO model in a simple way, by reducing the transport coefficients in reversed shear region. In particular, the Bohm contribution to the transport is allowed to go zero whenever the magnetic shear becomes negative. Similar approach of implementing the reversed magnetic shear and $\boldsymbol{E} \times \boldsymbol{B}$ flow shear effects has been adopted in some earlier simulations of internal transport barriers in tokamaks [38], and may suffice in an empirical transport model as well as for the purpose here.

Table 2.2: Global parameters for Ignitor simulation.

\begin{tabular}{ccccc}
\hline major radius & minor radius & elongation & triangluarity & toroidal field \\
$R(\mathrm{~m})$ & $a(\mathrm{~m})$ & $\kappa$ & $\delta$ & $B_{\phi}(\mathrm{T})$ \\
\hline 1.32 & 0.47 & 1.83 & 0.43 & 13 \\
\hline
\end{tabular}

In the simulation, we choose the global design parameters based on the specification given in Coppi et al. [13]. The relevant initial and boundary conditions for the transport equations are slected as following. The initial central temperatures for both ions and electrons are set to be $1 \mathrm{keV}$, and the 
initial central density is chosen to be $3.0 \times 10^{20} \mathrm{~m}^{-3}$ for both deuterium and tritium. During the heating process, the edge temperature increases from 0.3 $\mathrm{keV}$ at the beginning to $2.0 \mathrm{keV}$ in the end [15], while the edge density remains a constant value of $1.0 \times 10^{20} \mathrm{~m}^{-3}$ for both deuterium and tritium ion species.

Ignitor-f01

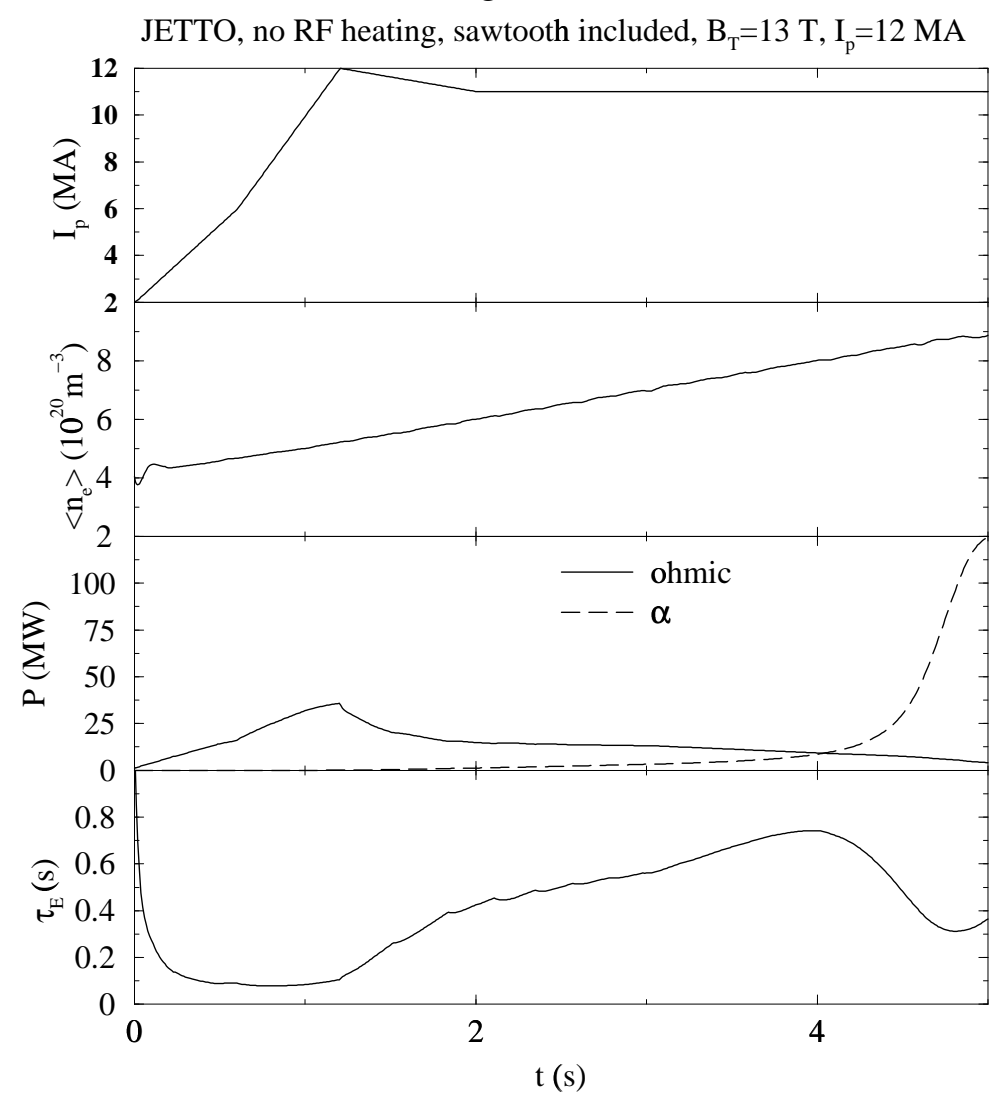

Figure 2.1: Time history of a) plasma current $I_{\mathrm{p}}, \mathrm{b}$ ) volume-average density $\left\langle n_{\mathrm{e}}\right\rangle$, c) Ohmic power $P_{\mathrm{oh}}$ and $\alpha$ power $P_{\alpha}$, and d) confinement time $\tau_{\mathrm{E}}$ in the reference simulation run f01 with peaked density profile.

The key procedure of the ohmic heating scheme in our simulation is 
the plasma current ramping. The time histories of plasma current and volume density for a reference simulation run f01 with peaked density profile are shown in Fig.2.1. The plasma current $I_{\mathrm{p}}$ is rapidly increased from its initial value during the first second, to its target maximum value of $12 \mathrm{MA}$ by the end of the ramping stage, drops to $11 \mathrm{MA}$, and then remains there for the rest of the heating process. With a fixed target value of plasma current and a fixed length of ramping period, the average current ramping rate is determined by the initial plasma current. We found that in order to have negative magnetic shear during the time evolution of magnetic flux surfaces, the initial plasma current should be approximately equal to or less than 4 MA. This corresponds to a threshold for the plasma current ramping rate of $7 \mathrm{MA} / \mathrm{s}$ approximately.

The rapid ramping of plasma current is accompanied by a gradual growth of electron volume-average density starting from $4.0 \times 10^{20} \mathrm{~m}^{-3}$ towards its target value of $9.0 \times 10^{20} \mathrm{~m}^{-3}$, controlled by neutral gas puffing from edge (Fig.2.1). For a given ramping rate of plasma current, there is an upper limit for density ramping rate above which the desired density profile peaking or plasma ignition would not occur. Too low a rate of density ramping, on the other hand, would also delay or even prevent ignition. A staged ramping scheme for volume averaged density with ramping rates carefully chosen between the two opposite ends could ensure the peaking of density profile as well as an early ignition, as, for example, in the reference case in Fig.2.1. 


\subsection{Simulation results}

With the transport model and simulation scheme presented in the previous section, peaked density profiles are obtained during early time stages (prior to ignition) of reference simulation run f01. In Fig.2.1 we show the optimal current and density ramp that has been found to date. During this time the ohmic power increases to its maximum of 39 MW as shown in Fig.2.1. The peak of the ohmic power reduced just at the end of the current ramp. The alpha power is at about $40 \mathrm{KW}$ at this time and will continue to increase rapidly as seen in Fig.2.1.

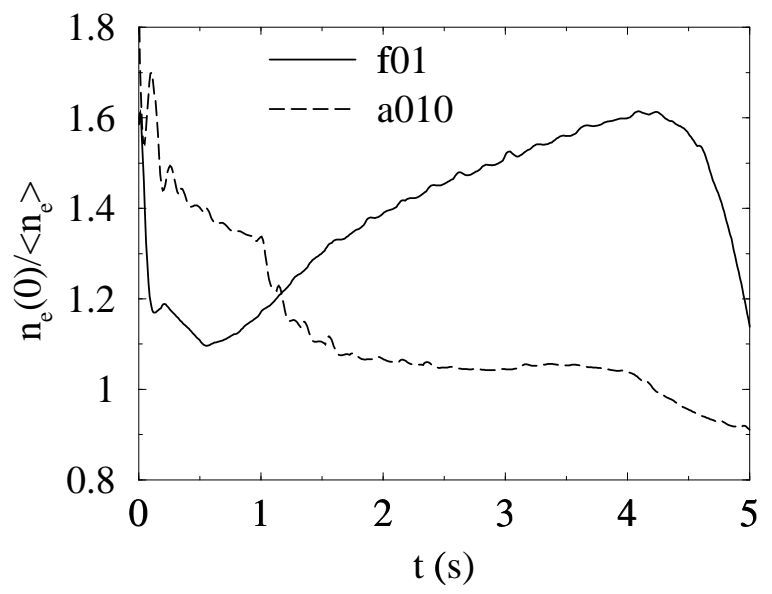

Figure 2.2: Comparison of time evolutions of density profile peakness $n_{\mathrm{e}}(0) /\left\langle n_{\mathrm{e}}\right\rangle$ for two simulation runs with different current ramping rates, i.e., f01 with fast rate and a010 with slow rate.

The radial electron density profiles at 6 representive time slices are plotted in Fig.2.3, in comparison with those of corresponding time slices in Fig.2.4 from an earlier simulation run a010 with less current ramping rate [15]. 


\section{Ignitor-f01}

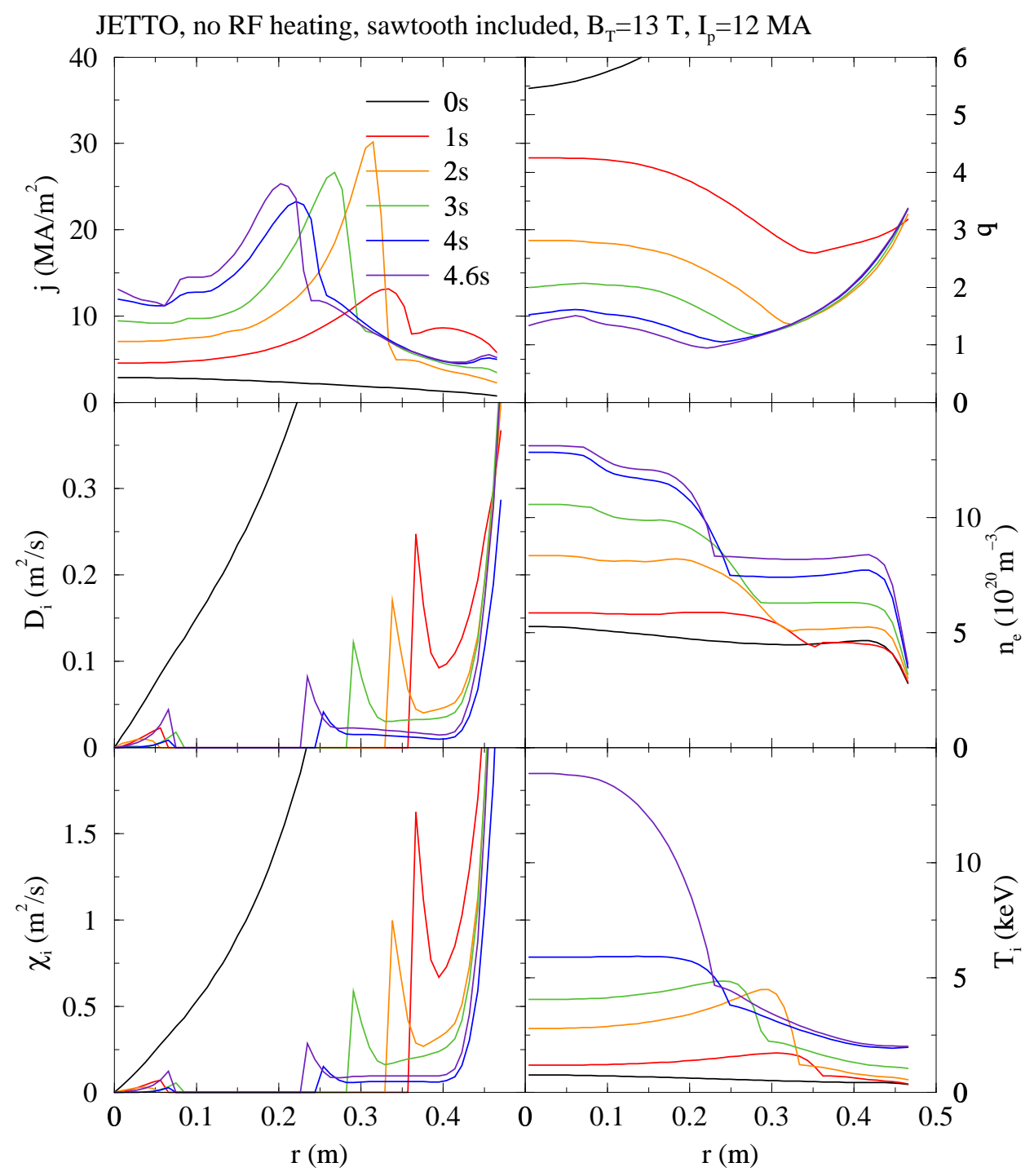

Figure 2.3: Radial profiles of a) plasma current density $j$, b) safety factor $q$, c) particle diffusivity $D_{i}$, d) electron density $n_{\mathrm{e}}$, e) ion thermal conductivity $\chi_{i}$, and f) ion temperature $T_{i}$, at six time slices $t=0,1,2,3,4$ and $4.6 \mathrm{~s}$ in the reference simulation run f01. 


\section{Ignitor-a10}

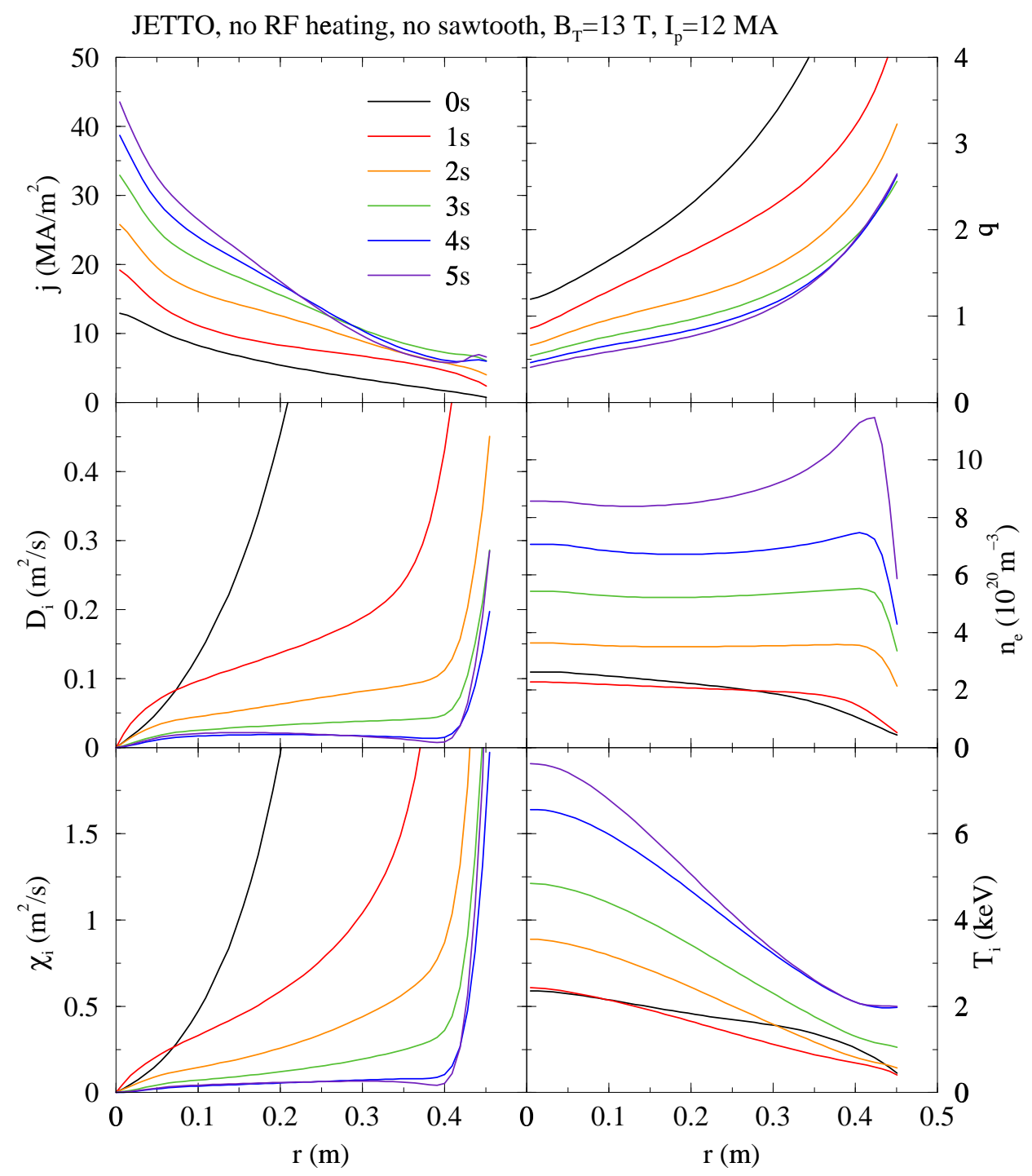

Figure 2.4: Radial profiles of a) plasma current density $j$, b) safety factor $q$, c) particle diffusivity $D_{i}$, d) electron density $n_{\mathrm{e}}$, e) ion thermal conductivity $\chi_{i}$, and $\mathrm{f}$ ) ion temperature $T_{i}$, at six time slices $t=0,1,2,3,4$ and $5 \mathrm{~s}$ in the reference simulation run a010. 


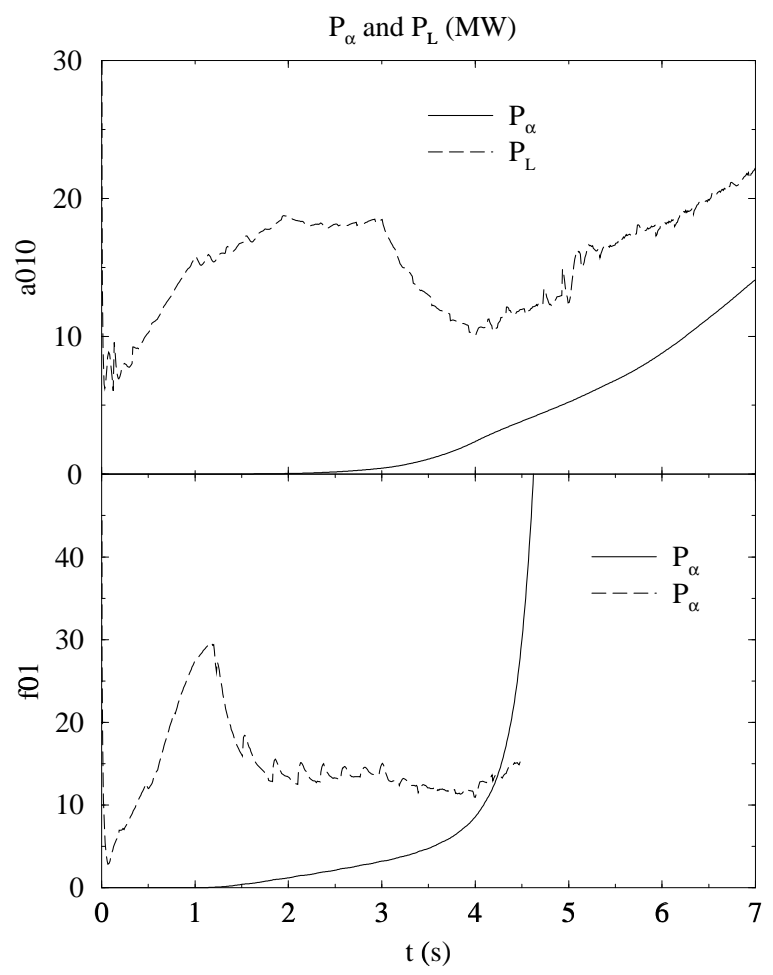

Figure 2.5: Time traces of alpha heating power $P_{\alpha}$ and the total confinement power loss $P_{\mathrm{L}}$ in the two simulation runs a010 and $\mathrm{f} 01$.

The peaking of density radial profile is evident in the reference case f01 at times prior to ignition, characterized by an inward moving internal transport barrier. Following Coppi et al. [14], we also use the ratio of the central density to the volume-averaged density $n_{\mathrm{e}}(0) /\left\langle n_{\mathrm{e}}\right\rangle$ to quantatize the peakness of the density profile. In Fig.2.2, we compare the time evolution of the density profile peakness $n_{\mathrm{e}}(0) /\left\langle n_{\mathrm{e}}\right\rangle$ of the two simulation cases. The correlation between the current ramping rate and the density profile peakness is apparant for time stages prior to ignition. 
The peaked density profiles seen in our simulation f01 are basically a direct consequence of the formation of internal transport barrier, produced indirectly by rapid current ramping through reversed magnetic shear. Such a mechanism of density peaking can be clearly demonstrated by the causal relations among the time-evolving radial profiles of several transport quantities, including plasma current density $j$, the safety factor $q$, the particle diffusivity $D_{\mathrm{i}}$, the electron density $n_{\mathrm{e}}$, the ion thermal conductivity $\chi_{\mathrm{i}}$, and the ion temperature $T_{\mathrm{i}}$ for the reference simulation run f01 in Fig.2.3. Initial plasma density and temperature radial profiles spread flatly. During the fast current ramping stage when poloidal magnetic flux sinks in from boundary, most of the plasma current accumulates around plasma edge if the current ramping rate beats the diffusion rate of the magnetic flux. As a result the safety factor profile is also dipped between the core and the edge as does that of ohmic heating power deposition. The reversed shear leads to the suppression of both particle and thermal transport in that region, forming steep gradients in both density and temperature profiles there, namely, an internal transport barrier. As the plasma is heated up, the reversed shear region and the transport barrier both move inward, resulting in the peaking of density profile. The timing and location of reversed shear region and the transport barrier are well correlated in profiles shown in Fig.2.3. As a comparison, the corresponding process in the earlier simulation run a010 is shown in Fig.2.4, where neither the reversed magnetic shear region or the internal transport barrier is present. The comparison for the plasma current ramping between a010 and f01 is shown in 
Fig. 2.6. The average current ramping rate for f01 is nearly three times of that for $\mathrm{a} 010$.

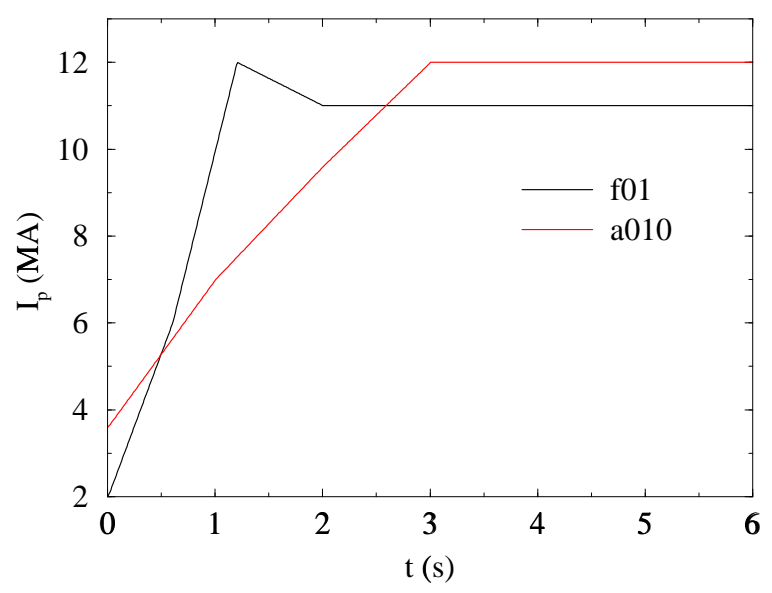

Figure 2.6: Comparison for time evolution of the plasma current between simulation runs f01 and a010.

The favorable effects of density profile peaking on ignition was demonstrated earlier in Coppi et al. [14]. However, our studies here differ from those of Coppi et al. in that the peaked density profiles in our transport simulations are produced dynamically by plasma current ramping instead of being prescribed artificially. As found in our simulations, plasma with peaked density profile during the heating process could reach ignition condition earlier than the time when sawtooth is triggered, which ensures the ignition. In Fig.2.5 we compare the time traces of alpha heating power $P_{\alpha}$ and the total confinement power loss $P_{L}$ for those simulation runs shown in Fig.2.2 with two different levels of density profile peaking due to different plasma current ramping rates. The ignition, defined as the moment when the $\alpha$-heating power $P_{\alpha}$ is balanced 
by the total thermal $\operatorname{loss} P_{L}$, can be seen to occur at $t=4.24 \mathrm{~s}$ in simulation f01 with density profile peaking, where the sawtooth oscillation starts at $t=4.34 \mathrm{~s}$. Moreover, the sawtooth is born in the middle of the minor radius. In simulation runs with less current ramping rate, sawtooth events kick in earlier than the time when the ignition conditions could be met, and it is born at the center of the plasma, and the $q \leq 1$ region has a larger volume, which prevent ignition from occuring. Fig. 2.7 shows the time evolution of the position of the $q=1$ surface for the two cases a010 and f01.

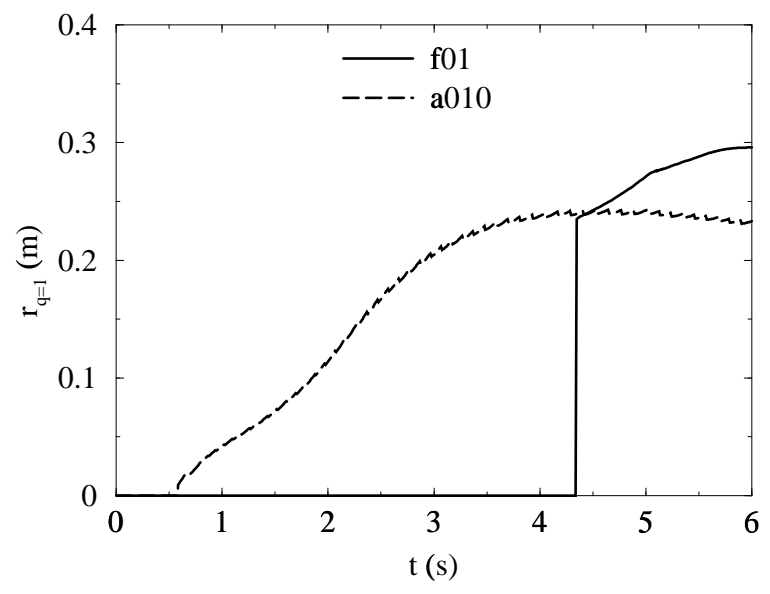

Figure 2.7: Comparison for time evolution of the position of $q=1$ surface between simulation runs f01 and a010.

\subsection{Summary}

The simulations reported in the previous section demonstrate a scheme of obtaining a peaked density profile during the ohmic heating process of Ignitor through the formation of internal transport barrier by rapid plasma current 
ramping. At the initial stage of ohmic heating, plasma current density would accumulate around the edge if the ramping rate of the total plasma current exceeds its inward diffusion rate. A minimum point in the profile of the safety factor $q$ appears where the plasma current density accumulates, forming a region with reversed magnetic shear. An internal transport barrier is thus produced in that region due to the suppression of fluctuation by reversed and weak magnetic shear there. Following the inward movement of the transport barrier as the plasma current sinks inside further, a peaked density as well as temperature profile is obtained. Peaked denisty and temperature profiles, as resulted from transport barrier, enable the Ignitor plasma to reach ignition condition earlier than the occurence of sawtooth events, hence ensure the achievement of ignition. Although the JETTO transport model is used in our simulations, such a density profile peaking scheme is in principle independent of the particular choice of transport model, since it derives from the instrinsic feature of ohmic heating process of Ignitor experiment and the universal mechanism of transport barrier formation due to reversed magnetic shear. Indeed with the standard multi-mode model built in the BALDUR code, the simulation results show the similar reversed magnetic shear $q$ profile and the density peaking effect. Fig. 2.8 shows the results from simulation with the multi-mode model transport model.

Moreover, ICRF auxiliary heating can be used to give additional noninductive current drive and keep the reversed magnetic shear for a longer time period. 


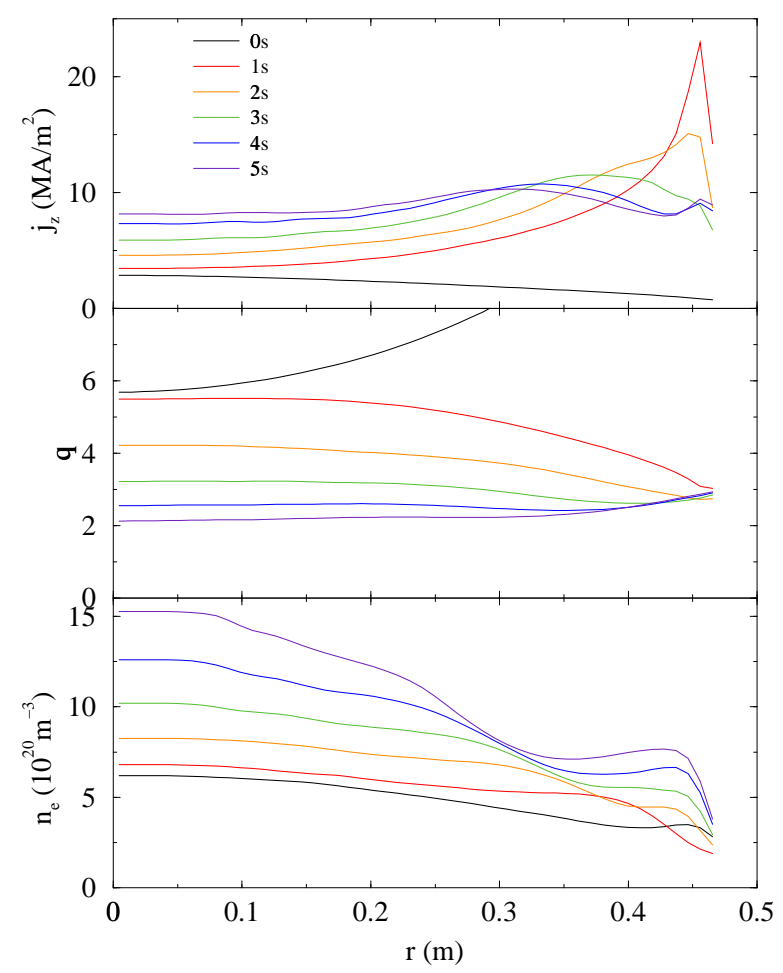

Figure 2.8: Current density $j$, safety factor $q$ and electron density $n_{\mathrm{e}}$ profiles at six time slices $t=0,1,2,3,4,5 \mathrm{~s}$. Results from BALDUR simulation with multi-mode model.

\subsection{Reversed shear experiments}

Reversed shear or optimized shear confinement enhancement has been achieved in TFTR [17], DIIID [18], JT60U [19], JET [20] and Tore Supra [21, 39], even though their scenarios for obtaining reversed shear are different.

In TFTR, a current is ramped up to $1.0 \mathrm{MA}$ with a ramp rate of 1.8 $\mathrm{MA} / \mathrm{s}$, then to $1.8 \mathrm{MA}$ with rate $0.4 \mathrm{MA} / \mathrm{s}$. particle transport drops by a factor of $\sim 40$ to the neoclassical level, and the ion thermal diffusivities drop 
much below the neoclassical values in the reversed shear region. The central electron density increases from $0.45 \times 10^{20} \mathrm{~m}^{-3}$ to $1.2 \times 10^{20} \mathrm{~m}^{-3}$ in $0.3 \mathrm{~s}$. The pressure profile has a peaking factor $p(0) /\langle p\rangle=8.0$, much larger than that of the L-mode, 3.3. In the reversed magnetic shear region, the central plasma pressure rises linearly in time to an MHD limit.

In DIIID, the reversed magnetic shear region is at $r / a \leq 0.5$, and has $20-30 \%$ of the total plasma volume. The thermal transport barrier appears $0.25 \mathrm{~s}$ after the reversed magnetic shear is created, and it is maintained for 0.6 s. The formation of the internal transport barrier produces a sudden increase in the central ion temperature, and the confinement time is increased to $H=1.4$ times of the previous value.

For JT-60U, the internal transport barrier extends up to $\sim 70 \%$ of the minor radius. The confinement is improved with a factor $H_{89 P}=\tau_{E} / \tau_{89 \mathrm{P}}=3.2$ compared with the ITER89-P scaling.

For Tore Supra, a current ramp rate of $1.6 \mathrm{MA} / \mathrm{s}$ is used, and the reversed shear is mentained for $2 \mathrm{~s}$. The internal transport barrier is obtained within $r / a \sim 0.3$. The confinement time enhancement factor is $H=1.4$ with the reversed magnetic shear.

\subsection{MHD stability}

Reversed magnetic shear can stabilize some drift-type microinstabilities, e.g., trapped electron modes, and MHD instabilities, e.g., ballooning 
modes, resistive tearing modes.

However, reversed magnetic shear may lead to other MHD instabilities, e.g., double tearing modes. This is because in the neighborhood of the point for the minimum $q$ value, there exist two points with the same mode numbers $m, n$. A previous study [40] shows that the non-linear evolution of tearing modes for equilibrium hollow current profiles may lead to a redistribution of the flux and field line reconnection as well as saturation.

It is thought that the instabilities caused by reversed magnetic shear is less dangerous than the sawtooth crash [14].

In the experiments for TFTR [17], the plasma is observed to be free of any coherent, low- $n$ or high- $n$ MHD activity throughout the reversed magnetic shear region.

In the experiments for DIIID [18], it is suggested that strong rotational shear may be important to stabilize the resistive instabilities caused by reversed magnetic shear. It is also suggested that the reversed magnetic shear along may not be sufficient. Sheared $\boldsymbol{E} \times \boldsymbol{B}$ flow associated with strong rotational shear is desirable.

For JT-60U [19], the plasma current, configuration and the beam injection are carefully controlled to achieve MHD stability with a significant bootstrap current.

Since the state of Ignitor is rather transient than steady, the calculation should be more complicated than that in [40], It is thought that the 
instabilities caused by the hollow current density profiles are less dangerous than the sawtooth crash. With the reversed magnetic shear, $q \leq 1$ region appears much later, has smaller volume, and starts from the middle of the radius, thus it seems to be much less dangerous. 
Appendix 


\section{Appendix A}

\section{Improved Maps for Anomalous Transport}

\section{A.1 Multi-ion map for anomalous transport}

For the case where many ions are distributed randomly in space, one can apply our map for each ion and introduce multi-ion-magneto-Coulomb map. This corresponds to the Lorentz model in statistical physics. To be simple, let us consider a series of ions distributed along a straight line parallel to the magnetic field. The locations of the ions are denoted by $z_{j}, 1 \leq j \leq N$, shown in Fig. A.1.

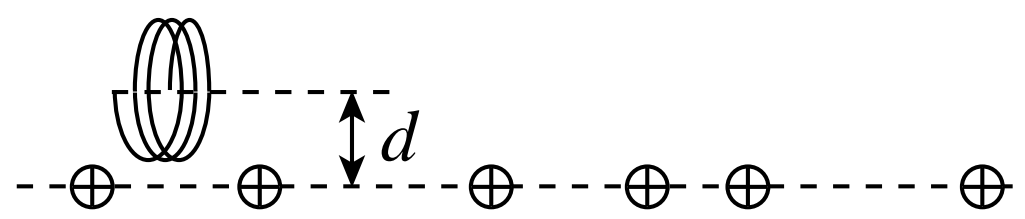

Figure A.1: Model for multi-ion map.

Then the magneto-Coulomb map is easily generalized as follows. which 
is also area-preserving.

$$
\left\{\begin{aligned}
v_{z}^{i+1}=v_{z}^{i}+ & \sum_{j=1}^{N} \frac{4\left(z_{i}-z_{j}+\pi v_{z}^{i}\right) \sqrt{(d-v)^{2}+\left(z_{i}-z_{j}+\pi v_{z}^{i}\right)^{2}}}{\left[d^{2}-v^{2}+\left(z_{i}-z_{j}+\pi v_{z}^{i}\right)^{2}\right]^{2}+4 v^{2}\left(z_{i}-z_{j}+\pi v_{z}^{i}\right)^{2}} \\
& \times E\left[\frac{-4 d v}{(d-v)^{2}+\left(z_{i}-z_{j}+\pi v_{z}^{i}\right)^{2}}\right] \\
z_{i+1}= & z_{i}+\quad \pi\left(v_{z}^{i}+v_{z}^{i+1}\right)
\end{aligned}\right.
$$

It is easily seen that this multi-ion map is also area-preserving.

\section{A.2 Anomaly in transport cofficients}

For ordinary diffusion, the variance of the distance is proportional to time. Intuitively, the trapping of the electron around the ion would give rise to the variance of the distance being proportional to $t^{\alpha}$, where $\alpha<1$. This happens when the average trapping time for the electron around one ion is infinity, which is caused by the long tail in the distribution of the trapping time. Long trapping time corresponds to the long distance between the electron and the ion. This can be seen from the Kepler period as a function of the radius. However, the existence of other ions will destroy this long-tail structure and give a finite average trapping time around one ion. The the only question is to ask in which region in the parameters $(d, v)$ the diffusivity is anomalous. 


\section{A.3 Derivation of convergent map}

In the chaotic scattering region, the magneto-Coulomb map gives a divergent $v_{z}$, when $z \rightarrow 0$ and $d \sim v$.

Here we construct a convergent map. The main idea is to use the pitch angle $\alpha$ instead of $v_{z}$ in the map, and $v_{z}$ is given by

$$
v_{z}=v \sin \alpha
$$

where the pitch angle $\alpha$ is the angle between the velocity $\boldsymbol{v}$ and the $x y$-plane. The auxiliary map can be written as

$$
\left\{\begin{aligned}
v\left(\sin \alpha^{i+1}-\sin \alpha^{i}\right) & =\frac{4{z^{\prime}}^{i} \sqrt{(d-v)^{2}+{z^{\prime}}^{2}}}{\left(d^{2}-v^{2}+{z^{\prime}}^{i^{2}}\right)^{2}+4 v^{2}{z^{\prime}}^{2}} E\left[\frac{-4 d v}{(d-v)^{2}+{z^{\prime}}^{2}}\right] \\
z^{\prime i+1}-z^{\prime i} & =2 \pi v_{z}^{i+1}
\end{aligned}\right.
$$

For small pitch angles, we can approximately write

$$
\left\{\begin{aligned}
v \tan \left(\alpha^{i+1}-\alpha^{i}\right) & =\frac{4 z^{\prime i} \sqrt{(d-v)^{2}+z^{i^{2}}}}{\left(d^{2}-v^{2}+z^{i^{2}}\right)^{2}+4 v^{2}{z^{\prime}}^{2}} E\left[\frac{-4 d v}{(d-v)^{2}+{z^{\prime}}^{2}}\right] \\
z^{\prime i+1}-z^{\prime i} & =2 \pi v_{z}^{i+1}
\end{aligned}\right.
$$

Then from these equations we derive a convergent map

$$
\left\{\begin{aligned}
\alpha^{i+1}-\alpha^{i} & =2 \tan ^{-1}\left(\frac{1}{2 v} \frac{4 z^{\prime} \sqrt{(d-v)^{2}+z^{i^{2}}}}{\left(d^{2}-v^{2}+{z^{\prime}}^{i^{2}}\right)^{2}+4 v^{2}{z^{\prime}}^{2}} E\left[\frac{-4 d v}{(d-v)^{2}+{z^{\prime}}^{2}}\right]\right) \\
z^{\prime i+1}-z^{\prime i} & ==2 \pi v \sin \alpha^{i+1}
\end{aligned}\right.
$$


It is area-preserving. Moreover, this improved map goes back to the original map (A.3) when (1.11) is satisfied. We note that the pitch angle can be changed up to $\pi$ in (A.5).

Since the map (A.5 is not accurate, in Fig.A.2, the phase space plots from the improved map and the numerical integration are compared for a slightly different initial conditions, i.e., $z_{0}=1.75$ for the improved map and $z_{0}=1.86$ for the numerical integration with the same $v_{z}^{0}=0, d=3$ and $v=1$. Qualitatively, the map shows the similar behavior as the equation of motion. 

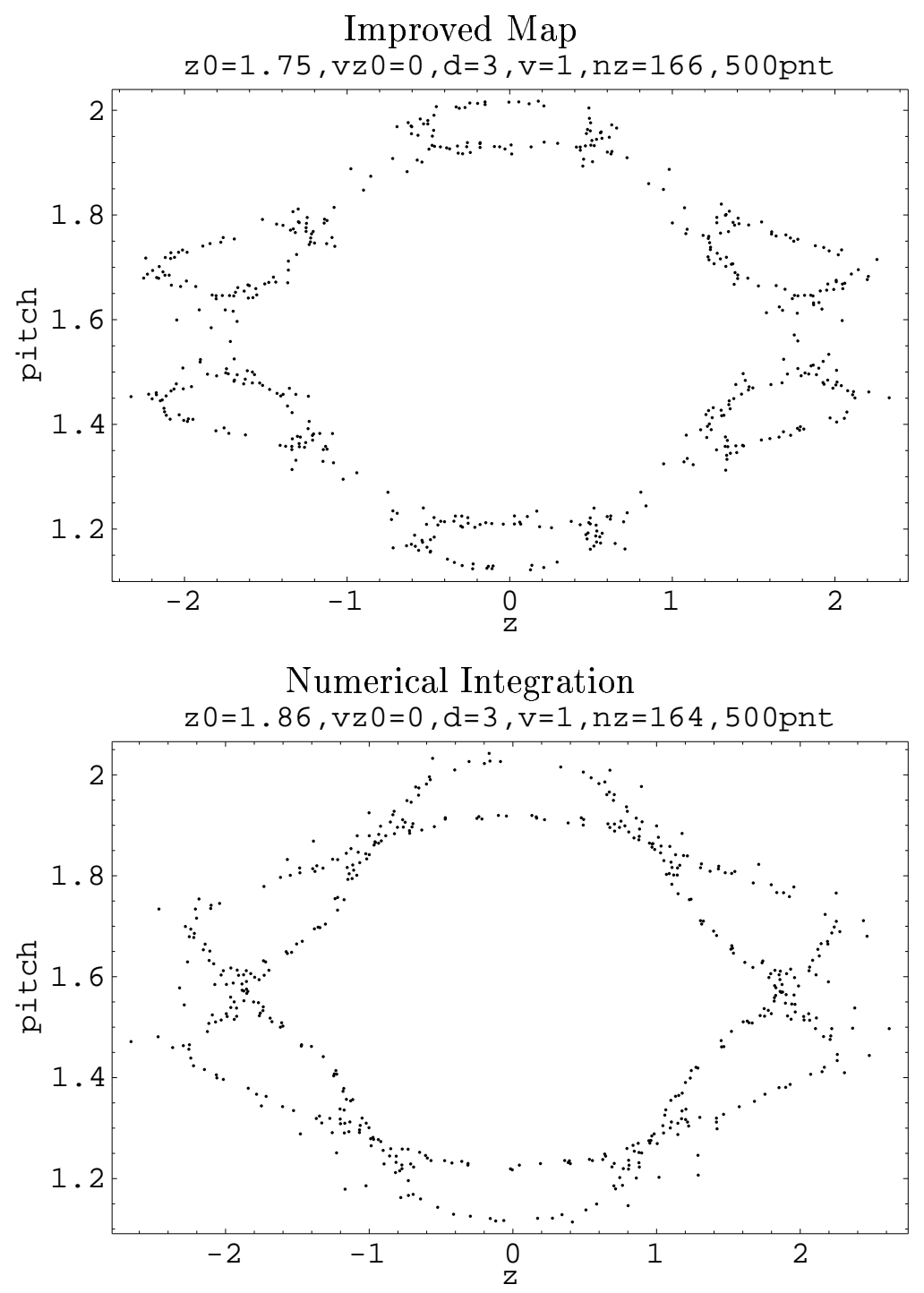

Figure A.2: $z^{0}=$ (Improved Map)1.75, (Numerical Integration)1.86, $v_{z}^{0}=0$, $v=1, d=3$. 


\section{Bibliography}

[1] J. B. Delos, S. K. Knudson, and D. W. Noid, Phys. Rev. A 30, 1208 (1984).

[2] G. Schmidt, E. E. Kunhardt, and J. L. Godino, Phys. Rev. E 62, $7512(2000)$.

[3] R. Gajewski, Physica 47, 975 (1970).

[4] J. B. Delos, S. K. Knudson, and D. W. Noid, Phys. Rev. A 28, 7 (1983).

[5] H. Hasegawa, M. Robnik, and G. Wunner, Prog. Theor. Phys. Suppl. 98, 198 (1989).

[6] B. Hu, W. Horton, C. Chiu, and T. Petrosky, Phys. Plasmas 9, 1116 (2002).

[7] H. Friedrich and D. Wintgen, Phys. Rep. 183, 37 (1989).

[8] M. Robnik, J. Phys. A, Math. Gen. 14, 3195 (1981).

[9] C. L. Siegel and J. K. Moser, Lectures on Celestial Mechanics, SpringerVerlag, 1971.

[10] T. Petrosky, Phys. Lett. A 117, 328 (1986). 
[11] T. C. Killian et al., Phys. Rev. Lett. 83, 4776 (1999).

[12] J. Godino, E. Kunhardt and W. Carr, APS-DPP meeting poster QP1.97, 2001.

[13] B. Coppi et al., Critical physics issues for ignition experiments: Ignitor, MIT (RLE) Report PTP 99/06.

[14] B. Coppi, M. Nassi, L. E. Sugiyama, Physica Scripta 45, 112 (1992).

[15] W. Horton et al., Nucl. Fusion 42, (2000).

[16] F. X. Söldner et al., Phys. Rev. Lett. 61, 1105 (1988).

[17] F. M. Levinton et al., Phys. Rev. Lett. 75, 4417 (1995).

[18] E. J. Strait et al., Phys. Rev. Lett. 75, 4421 (1995).

[19] S. Ishida et al., Phys. Rev. Lett. 79, 3917 (1997).

[20] C. Gormezano et al., Phys. Rev. Lett. 80, 5544 (1998).

[21] G. T. Hoang et al., Phys. Rev. Lett. 84, 4593 (2000).

[22] W. Horton et al., Phys. Plasmas 5, 3910 (1998).

[23] J. M. Kwon et al., Phys. Plasmas 7, 1169 (2000).

[24] M. Erba et al., Nucl. Fusion 38, 1013 (1998)

[25] B. Hu et al., IFS Report \#943, 2002. 
[26] C. E. Singer et al., Computer Physics Communications 49, 275 (1988).

[27] G. Bateman, Simulation of transport in tokamaks, Springer-Verlag, 1991.

[28] R. J. Goldston and P. H. Ruthorford, Introduction to plasma physics, Institute of Physics Publishing, 1995.

[29] J. Wesson, Tokamaks, Oxford University Press, Inc., 1997.

[30] G. Bateman et al., Phys. Plasmas 5, 1793 (1998).

[31] A. Taroni et al., Plasma Phys. Control. Fusion 36, 1629 (1994).

[32] M. Erba et al., Plasma Phys. Control. Fusion 37, 1249 (1995).

[33] M. Erba et al., Plasma Phys. Control. Fusion 39, 261 (1997).

[34] M. Erba et al., Subroutine for computing particle and energy fluxes using the mixed transport model, unpublished, 1999.

[35] C. Kessel et al., Phys. Rev. Lett. 72, 1212 (1994).

[36] M. A. Beer et al., Phys. Plasmas 4, 1792 (1997).

[37] Y. Kishimoto et al., Plasma Phys. Control. Fusion, 41, A663 (1999).

[38] V. Parail et al., Nucl. Fusion 39, 429 (1999).

[39] X. Litaudon et al., Plasma Phys. Control. Fusion 38, 1603 (1996). 
[40] B. Carreras, H. R. Hicks and B. V. Waddell, Nucl. Fusion 19, 583 (1979). 


\section{Vita}

Bo Hu was born in Beijing, China, on October 9, 1971, the son of Yuli $\mathrm{Hu}$ and Shaojia Tan. He received the Bachelor of Science degree in physics from Peking University in China and came to the United States and entered the physics Ph.D. program at the University of Texas at Austin in Fall 1995. He married Tong Zhang on August 1, 1997. After spending three years in the field of particle physics, he realized that he had made no progress in his research, although he might love research in particle physics. Then he received a Master of Arts degree in particle physics, then switched to plasma physics and entered the Institute for Fusion Studies. Isaac $\mathrm{Hu}$, his son, was born on February 5, 2001.

Permanent address: $1642 \mathrm{~W}$ 6th St Apt D

Austin, Texas 78703

This dissertation was typeset with $\mathrm{LT}_{\mathrm{E}} \mathrm{X}^{\dagger}$ by the author.

\footnotetext{
${ }^{\dagger} \mathrm{L}_{\mathrm{A}} \mathrm{T} \mathrm{X}$ is a document preparation system developed by Leslie Lamport as a special version of Donald Knuth's $\mathrm{T}_{\mathrm{E}} \mathrm{X}$ Program.
} 